\title{
8
}
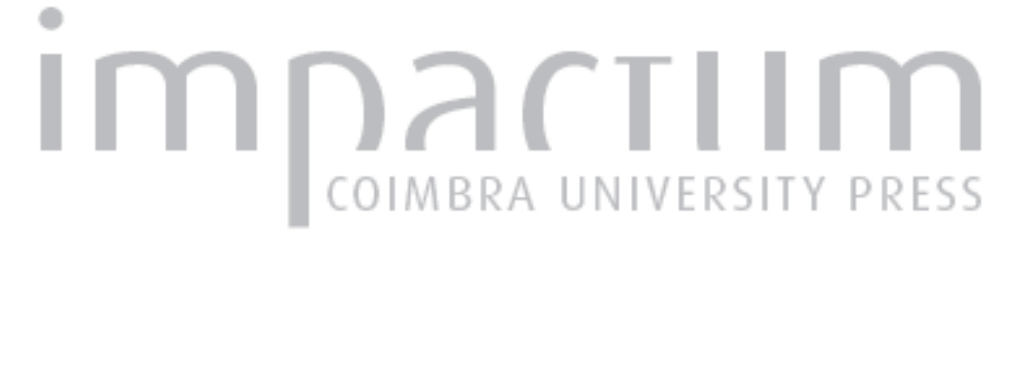

\section{Princípios de Medicina de catástrofe em revisão a partir de Fukushima} \author{
$\begin{array}{ll}\text { Autor(es): } & \text { Silva, Rita Marques da; Campos, Paulo; Reis, Ana Mafalda; Bandeira, } \\ \text { Romero }\end{array}$
}
Publicado por: Associação Portuguesa de Riscos, Prevenção e Segurança; Imprensa da Universidade de Coimbra

URL

persistente:

URI:http://hdl.handle.net/10316.2/38075

DOI:

DOI:http://dx.doi.org/10.14195/1647-7723_22_19

\section{Accessed : $\quad$ 26-Apr-2023 08:50:19}

A navegação consulta e descarregamento dos títulos inseridos nas Bibliotecas Digitais UC Digitalis, UC Pombalina e UC Impactum, pressupõem a aceitação plena e sem reservas dos Termos e Condições de Uso destas Bibliotecas Digitais, disponíveis em https://digitalis.uc.pt/pt-pt/termos.

Conforme exposto nos referidos Termos e Condições de Uso, o descarregamento de títulos de acesso restrito requer uma licença válida de autorização devendo o utilizador aceder ao(s) documento(s) a partir de um endereço de IP da instituição detentora da supramencionada licença.

Ao utilizador é apenas permitido o descarregamento para uso pessoal, pelo que o emprego do(s) título(s) descarregado(s) para outro fim, designadamente comercial, carece de autorização do respetivo autor ou editor da obra.

Na medida em que todas as obras da UC Digitalis se encontram protegidas pelo Código do Direito de Autor e Direitos Conexos e demais legislação aplicável, toda a cópia, parcial ou total, deste documento, nos casos em que é legalmente admitida, deverá conter ou fazer-se acompanhar por este aviso. 


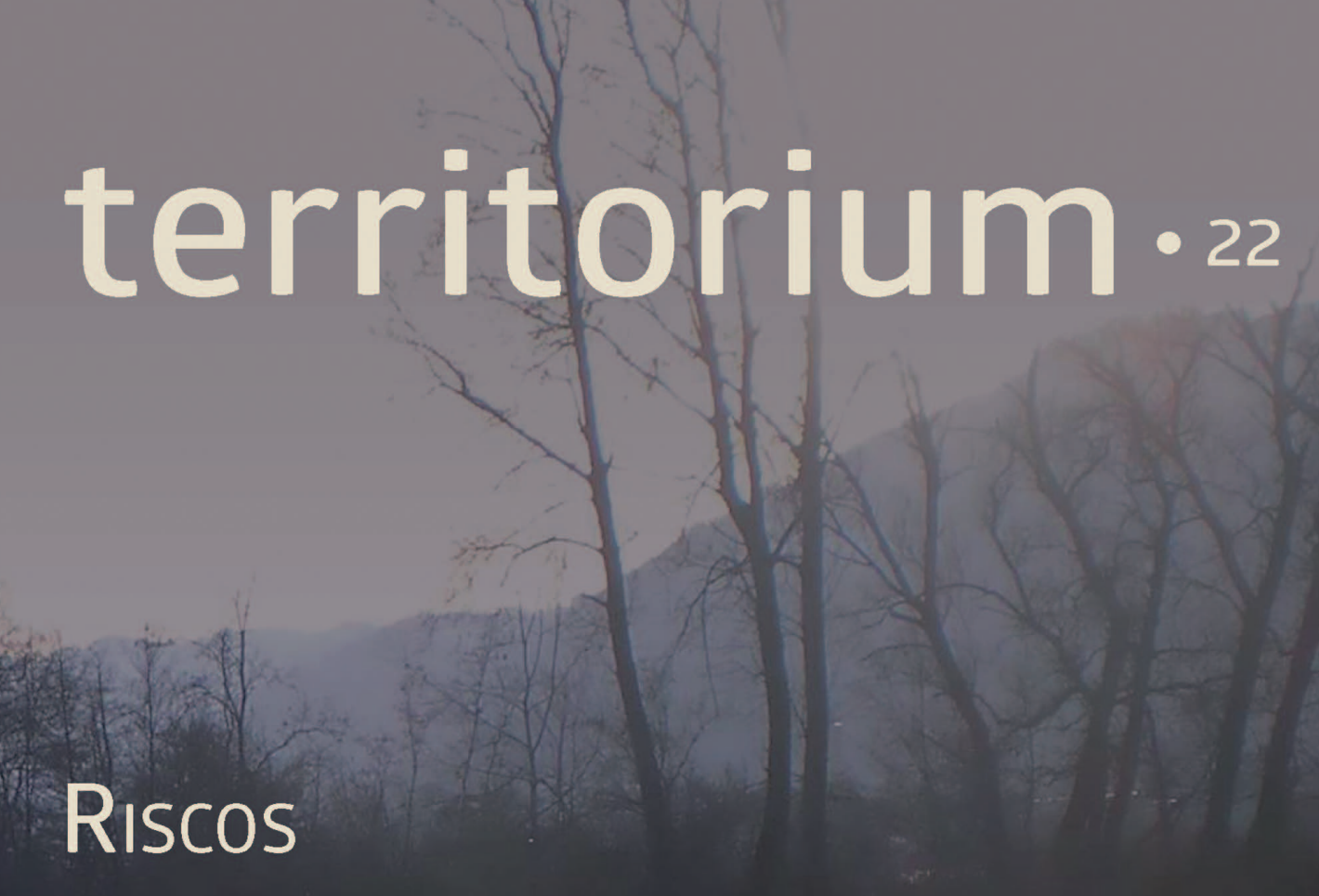

\section{TERRITÓRIOS DE CONVERGÊNCIA}

- Imprensa da Universidade de Coimbra Associação Portuguesa de:Riscos, Prevenção e Segurança 


\section{PRINCÍPIOS DE MEDICINA DE CATÁSTROFE EM REVISÃO A PARTIR DE FUKUSHIMA*}

\section{DISASTER MEDICINE PRINCIPLES UNDER REVIEW FROM FUKUSHIMA}

Rita Marques da Silva

Instituto de Ciências Biomédicas Abel Salazar

Universidade do Porto

ritasuzana.gms@gmail.com

Paulo Campos

Instituto de Ciências Biomédicas Abel Salazar

Universidade do Porto

pacampos@netcabo.pt / paulocampos@inem.pt
Ana Mafalda Reis

Instituto de Ciências Biomédicas Abel Salazar Universidade do Porto docmaf@sapo.pt

Romero Bandeira

Instituto de Ciências Biomédicas Abel Salazar Universidade do Porto hmedcat@icbas.up.pt

\section{RESUMO}

Pelas suas características inéditas, consequências além do expectável e dimensão mundial, é abordada a catástrofe que ocorreu a 11/03/2011 em Fukushima, Japão. Através da revisão de conceitos básicos, analisa-se a evolução dos acontecimentos, protocolos de atuação médica em socorro a multivítimas com deslocação obrigatória de populações inteiras e as consequências a curto, médio e longo prazo dos efeitos radiológicos, saúde pública, problemas sociais e psicológicos, não só para as populações atingidas mas também para os interventores, numa perspetiva de minimizar as taxas de morbi e mortalidade nas gerações futuras.

Palavras-chave: Tsunami, energia nuclear, socorro a multivítimas, telemedicina, saúde pública.

\section{ABSTRACT}

Due to its unique characteristics, consequences beyond the expected and global dimension, we discuss disaster that occurred 11/03/2011 in Fukushima, Japan. A review of basic concepts, analyzes the developments, medical action protocols help the multivictms with compulsory displacement of entire populations and the consequences in the short, medium and long-term radiological effects, public health, social and psychological problems not only for the people affected but also for intervening in a perspective of minimizing morbidity and mortality rates in future generations.

Keywords: Tsunami, nuclear power, the multi victims first aid, telemedicine, public health.

\section{RESUMEN}

Principios de Medicina de Desastres en revisión, después de Fukushima - Debido a sus características únicas, con consecuencias más allá de las esperadas y con dimensión global, se discute el desastre ocurrido el 11/03/2011 en Fukushima, Japón. Mediante la revisión de conceptos básicos, se analiza tanto la evolución de los acontecimientos, como los protocolos de acción médica en rescate con múltiples víctimas y desplazamiento obligatorio de poblaciones enteras y, aún, las consecuencias a corto, mediano y largo plazo, de los efectos radiológicos en la salud pública, los problemas sociales y psicológicos no sólo en las personas afectadas, sino también en las que han intervenido, con una perspectiva de minimizar las tasas de morbilidad y mortalidad en las generaciones futuras.

Palabras clave: Tsunami, energía nuclear, ayuda a múltiples víctimas, telemedicina, salud pública.

\section{RÉSUMÉ}

Principes de médecine de catastrophe revue de Fukushima - Grâce à ses caractéristiques uniques, conséquences au-delà de la dimension attendue et mondial, nous discutons le désastre survenu au 11/03/2011 à Fukushima, au Japon. Un examen des concepts de base, analyse les développements, les protocoles d'action médicaux à la rescousse pour multi-vítimas victimes avec déplacement obligatoire de populations entières et les conséquences dans les effets radiologiques à court, moyen et long terme, la santé publique, les problèmes sociaux et psychologiques non seulement pour les personnes concernées mais aussi pour intervenir dans une perspective de réduire la morbidité et de mortalité dans les générations futures.

Mots-clé: Tsunami, l'énergie nucléaire, aider les multi-victimes, la télémédecine, la santé publique.

* O texto deste artigo corresponde a uma comunicação apresentada no III Congresso Internacional, I Simpósio Ibero-Americano e VIII Encontro Nacional de Riscos, tendo sido submetido em 12-01-2015, sujeito a revisão por pares a 30-04-2015 e aceite para publicação em 17-07-2015.

Este artigo é parte integrante da Revista Territorium, n. ${ }^{\circ} 22,2015,{ }^{\circ}$ RIscos, ISSN: 0872-8941. 


\section{Introdução}

A noção de Medicina de Catástrofe surge-nos como uma prioridade numa emanação dos tempos que correm. Não se trata de uma especialidade ou de uma competência que tenha surgido por diferenciação programada ou específica, única e exclusivamente de âmbito médico, mas sim pela aglutinação de situações de extrema gravidade para pessoas e bens que a sociedade neste século $X X$ desenvolveu ou criou e que os médicos para as resolverem se vêm obrigados a lançar mão de recursos sociais polivalentes, pois caso contrário a resposta a essas situações de crise não será minimamente suficiente. Estas circunstâncias fazem com que a Medicina de Catástrofe se paute por métodos e processos específicos e diferentes da Medicina de Urgência habitual (R. Bandeira, 2008).

Segundo o $3^{\circ}$ artigo da Lei de Bases da Proteção Civil, lei $n^{\circ}$ 27/2006 de 3 de Julho aprovada pela Assembleia da República Portuguesa: "Acidente grave é um acontecimento inusitado com efeitos relativamente limitados no tempo e no espaço, suscetível de atingir as pessoas e outros seres vivos, os bens ou o ambiente. Catástrofe é o acidente grave ou a série de acidentes graves suscetíveis de provocarem elevados prejuízos materiais $e$, eventualmente, vitimas, afetando intensamente as condições de vida e o tecido sócio-económico em áreas ou na totalidade do território nacional."

A definição da Organização Mundial de Saúde (OMS) é clara: catástrofe é qualquer acontecimento que cause estragos, destabilização económica, perda de vidas humanas e deterioração de saúde e dos serviços de saúde, a uma escala tal, que justifique uma mobilização excecional de auxílios vindos de fora da comunidade ou da zona atingida.

O conceito de catástrofe muito para além da sua definição baseia-se em três componentes: afluxo intenso de vítimas; destruições de ordem material e desproporcionalidade acentuada entre os meios humanos e materiais de socorro e as vítimas a socorrer (R. Bandeira, 2008).

Os vários tipos de classificação são fundamentalmente obtidos com base na etiologia ou nas consequências de uma catástrofe. Uma classificação mais recente e com maior relevância no âmbito da Medicina de Catástrofe é a que diz respeito fundamentalmente à seguinte análise paramétrica, baseada nos princípios de R. Noto et al., 1994: quanto aos efeitos sobre a comunidade (simples e complexa); à causa (de aparecimento rápido e de instalação longa); à duração do fator desencadeante: catástrofes curtas (<1hora), catástrofes médias (<24horas), catástrofes longas (>24horas); à duração do salvamento: catástrofes curtas (<6horas), catástrofes médias (>6horas e <24horas), catástrofes longas (>24horas); à extensão geográfica (raio $<1 \mathrm{~km}$, raio de 1 a $100 \mathrm{~km}$, raio $>100 \mathrm{~km}$ ); à região (urbanas e rurais); às vítimas: moderada (número de vítimas entre 25 e 99), média (número entre 100 e 999, mas 50 a 250 hospitalizados), maior (número de vítimas superior a 1000 e mais de 250 hospitalizados); à patologia apresentada; à evacuação (R. Bandeira, 2008).

Segundo A. M. Silva (2009), que cita R. Bandeira, a classificação mais atual e que melhor se adequa à realidade nacional distingue 4 situações:

- Acidente Catastrófico de Efeito Limitado (ACEL) - acidente limitado no tempo (algumas horas), no espaço (confinado ao local em que se desencadeou ou nas proximidades), e desprovido em princípio de risco evolutivo, podendo ser resolvido na maioria das vezes com os recursos previamente existentes. (Exemplos: acidentes de avião, de comboio, ou similares);

- Acidente Catastrófico de Efeito Major (ACEM) acidente de abrangência alargada, quer em termos de implicados, quer no seu impacto social e político. Número de vítimas entre 100 a 1000;

- Catástrofe - maior abrangência em termos de território atingido, com implicações graves na rede de distribuição de água, eletricidade, gás e saneamento. Número de vítimas superior a 1000;

- Mega Catástrofe - evento de âmbito alargado geograficamente, com um número de vítimas que excede sempre as 3000, caracterizase por uma destruição massiva numa ampla região territorial. (Exemplo: Tsunami de 26 de Dezembro de 2004 no Sudoeste Asiático, que vitimou 230.000 pessoas).

\section{Catástrofes Naturais}

Apesar de todo o avanço científico e tecnológico algumas questões continuam insolúveis. Assim não há relação clara entre a intensidade e a magnitude de um sismo; de três em três dias aparece um sismo potencialmente perigoso (magnitude 6 em Escala de Richter) em qualquer ponto do planeta; não é possível preverem-se seguramente os tremores de terra (C. Allégre, 1993; R. Bandeira, 2008; J. P. Rothé, 1946).

Além dos muitos benefícios que o oceano traz, este tem sérios riscos sísmicos e meteorológicos. Os ciclones tropicais (também conhecidos como furacões ou tufões) e Tsunamis representam os perigos mais poderosos e destrutivos do mar (J. H. E. Cartwright e H. Nakamura, 2008).

Tsunami é o resultado de uma grande quantidade de água que é deslocada num curto espaço de tempo. Isto ocorre tipicamente com movimentos de terra 
devido a terremotos submarinos (maremotos), erupções vulcânicas ou deslizamentos de terra no oceano, num mar ou num lago grande. Um segundo mecanismo é uma grande quantidade de material subitamente a entrar na água, quer a partir de um deslizamento de terra em terra que termina na água, quer a partir do impacto de um meteorito. Em ambos os casos, o deslocamento súbito de uma grande massa de água inicia uma onda, ou várias seguidas, que pode ter um comprimento extremamente longo de centenas de quilómetros (J. H. E. Cartwright e H. Nakamura, 2008). Cerca de $90-95 \%$ dos Tsunamis são causados por grandes terramotos (habitualmente de magnitude 7.5 (M. E. Keim, 2006) na escala de Richter (C. F. Richter, 1935) ou maior) em zonas de subducção (onde uma placa tectónica desliza sobre a outra) (M. E. Keim, 2006).

Vários termos em inglês foram abandonados a favor da utilização generalizada da palavra japonesa Tsunami, significando ondas (nami) a quebrar em cima de um porto (tsu) (J. H. E. Cartwright e H. Nakamura, 2008).

Tsunamis têm o potencial de causar um enorme impacto com milhões de mortes. De 1895 a 1995, foram registados 454 Tsunamis no Pacífico, os 94 mais mortais originaram 51.000 mortes. 0 Tsunami de 2004 no Oceano Índico matou perto de 300.000 pessoas e afetou mais de 2.000.000 em doze nações (M. E. Keim, 2006).
Os efeitos na saúde dos ciclones e dos Tsunamis não devem ser subestimados (TABela I e II). Além da saúde pública e das consequências médicas destes desastres, o impacto social, cultural e psicológico dos ciclones e Tsunamis tem um enorme e duradouro efeito pelo mundo, e um efeito direto sobre o desenvolvimento humano em geral (M. E. Keim, 2006).

\section{Catástrofes Nucleares e Radiológicas}

Emergências nucleares, radiológicas, biológicas e químicas (NRBQ) ocorrem por exposição ocupacional, incêndio, explosão, libertação de tóxicos ou químicos perigosos, agentes de guerra biológica, e são causadas por ignorância, negligência, incompetência, acidente ou intencionais (R. K. Sharma, 2010).

O surgimento do terrorismo, em particular o de Estado, a proliferação de armas químicas/biológicas, a disponibilidade de materiais e conhecimento científicos de armas, e o recente aumento de ataques menos discriminativos, apontam para uma probabilidade crescente de incidentes de vítimas em massa (R. K. Sharma, 2010).

A ameaça NRBQ, quer seja por produção de armas de destruição massiva por parte de um Estado, ou por terrorismo, contribui para um clima de insegurança

TABela I - Consequências na Saúde Pública dos Ciclones e Tsunamis em Cyclones, Tsunamis, and Human Health.

TABLE I - Impact on Public Health of cyclones and tsunamis in Cyclones, Tsunamis, and Human Health.

\begin{tabular}{|c|c|c|}
\hline Consequence & Cyclones & Tsunami \\
\hline Death rates & $\begin{array}{l}\text { Developed nations - Low } \\
\text { Developing nations-High }\end{array}$ & High \\
\hline Severe injuries (\% among survivors) & Few & Few to moderate \\
\hline Loss of clean water & Widespread & Focal to widespread \\
\hline Loss of shelter & Widespread & Focal to widespread \\
\hline Loss of personal and household goods & Widespread & Focal to widespread \\
\hline Major population movements & Rare & Rare \\
\hline Loss of routine hygiene & Widespread & Focal to widespread \\
\hline Loss of sanitation & Widespread & Focal to widespread \\
\hline Disruption of solid waste & Widespread & Focal to widespread \\
\hline Public concern for safety & High & High \\
\hline Increased pests and vectors & Widespread & Focal to widespread \\
\hline Loss and/or damage of health care system & Widespread & Focal to widespread \\
\hline Worsening of existing chronic illnesses & Widespread & Focal to widespread \\
\hline Loss of electricity & Widespread & Focal to widespread \\
\hline Toxic exposures & Possible & Possible \\
\hline Food scarcity & Common in low-lying remote islands & Common in early stages only \\
\hline
\end{tabular}


e preocupação nos países ocidentais (Alferes RC F. Domingos, 2013). Esta ameaça, que durante muito tempo passou despercebida, exige atualmente o estabelecimento de sistemas de vigilância e de mecanismos de resposta e constitui uma preocupação específica dos Órgãos de Estado, das Forças Armadas e da Sociedade em geral (Alferes RC F. Domingos, 2013).

Após o acidente de Chernobyl em 1986, foi amplamente reconhecido que os acidentes nucleares podem ter consequências sobre amplas áreas geográficas, podendo facilmente tornar-se um evento internacional com necessidade de resposta ao mesmo nível (F. Ugletveit e W. Molhoek, 2004).

\section{Dados e Metodologia}

Partindo dos conceitos básicos de como se aborda uma situação de multivítimas/catástrofe, foi realizada uma pesquisa bibliográfica em sites de publicações científicas, incluindo Revistas específicas de Medicina de Catástrofe e Cuidados Pré-hospitalares e Saúde Pública. Foram consultados livros com os conceitos primordiais da área da triagem em catástrofe como "Medicina de Catástrofe, da Exemplificação Histórica à latroética" e também teses de candidatura ao grau de Mestrado em Medicina de Catástrofe e Doutoramento em Ciências Médicas.

TABELA II - Frequência Relativa Estimada de Efeitos na Saúde Associados a Ciclones e Tsunamis em Cyclones, Tsunamis, and Human Health.

TABLE II - Frequency Relative Estimated Effects on Health Associates to cyclones and Tsunamis in Cyclones, Tsunamis, and Human Health.

\begin{tabular}{|l|c|c|}
\hline Type of morbidity & Cyclone & Tsunami \\
Crush injury & ++ & ++ \\
Head injury & + & ++ \\
Asphyxiation & + & ++ \\
Isolated bone injury & + & ++ \\
Skin soft tissue injury & + & ++ \\
Burns & + & 0 \\
Drowning & ++ & ++ \\
Asthma/emphysema & + & 0 \\
Hypothermia & 0 & + \\
Epidemics & 0 & + \\
Starvation & + & + \\
Aspiration pneumonia & 0 & + \\
Tetanus & + & + \\
Wound infections & + & + \\
Psychologicalilness & + & + \\
\hline
\end{tabular}

Fonte: The Key Role of Preparedness, Oceanography, 2006.
Para abordar o caso específico de Fukushima consultei vários artigos de revisão e estudos prospetivos e caso controlo para interpretar os acontecimentos e suas consequências e retirar conclusões sobre este evento, seu impacto mundial e relevância médica.

\section{Desenvolvimento}

\section{Acidente em Fukushima}

\section{Como Aconteceu}

É ainda muito cedo para compreender completamente o que aconteceu na estação de energia nuclear DaiIchi de Fukushima (FNP-I), Japão. No entanto, as informações disponíveis irão permitir-nos saber se o pior acidente nuclear desde Chernobyl poderia ter sido evitado. Além do desastre natural e risco técnico, a falha do sistema de regulação nuclear do Japão também foi culpado pelo desastre nuclear de Fukushima (Q. Wang e X. Chen, 2011).

Primeiramente autorizada em 1971, a instalação consiste em 6 reatores de água em ebulição. Tais reatores usam o calor produzido pela fissão nuclear para ferver a água (pressurizado a cerca de $75 \mathrm{~atm}$ a uma temperatura de cerca de $285^{\circ} \mathrm{C}$ ) para produzir vapor. Quando operacional, a estação providencia um total de 4,7 gigawatts de energia elétrica, tornando-se numa das 15 maiores instalações de energia nuclear no mundo (L. T. Dauer et al., 2011).

A 11 de Março de 2011, um terremoto de magnitude 9 ocorreu no fundo do mar (a $24 \mathrm{~km}$ de profundidade (T. Tanaka, 2012)) a cerca de $130 \mathrm{~km}$ ao largo da costa nordeste da principal ilha do Japão, ilha de Honshu (K. Ono, 2012), e consequentemente um Tsunami que atingiu uma altura sem precedentes de cerca de 14 metros (L. T. Dauer L. T. et al., 2011) (fig. 1).

A FNP-I localiza-se na costa a cerca de $200 \mathrm{~km}$ a sudoeste do epicentro. No momento do terremoto, três dos seis reatores estavam em operação. Apesar de terem sofrido um abalo de uma magnitude superior à esperada quando projetadas, o sistema de segurança desligou os reatores automaticamente. No entanto, uma torre da energia colapsou, interrompendo a fornecimento de eletricidade à estação. Após 40 minutos do terremoto, o enorme Tsunami embateu nos edifícios das turbinas que continham os geradores a diesel para o abastecimento em situações de emergência (TABELA III). Devido à perda de toda a energia elétrica, os sistemas de arrefecimento dos reatores ficaram paralisados (K. Ono, 2012).

Em cada um destes acontecimentos, bem como durante as operações para aliviar a pressão do vapor, foi libertada no meio ambiente radioatividade significativa. 


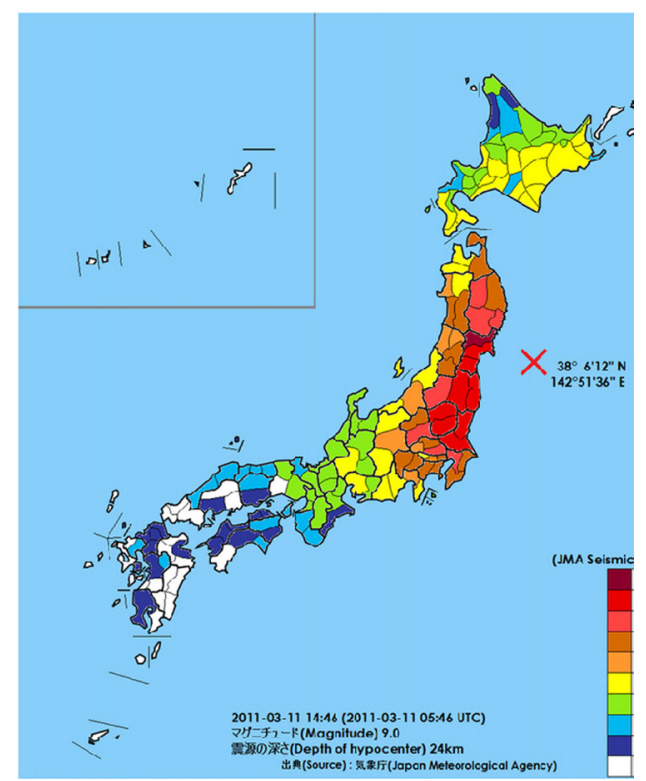

Fig. 1 - Epicentro do terremoto na costa do Oceano Pacífico de Tohoku e a distribuição da intensidade sísmica, em Characteristics and problems of fires following the Great East Japan earthquake in March 2011, 2012.

Fig. 1 - Epicenter of the earthquake off the coast of the Tohoku Pacific Ocean and Distribution of seismic intensity, in Features and problems of fires following the Great East Japan Earthquake in March 2011, 2012.
A fig. 2 mostra os resultados das medições aéreas de deposição de césio total após o acidente nas instalações de FNP - I com base em dados compilados pelo Departamento de Energia dos EUA, a Agência Nacional de Segurança Nuclear americana e as autoridades japonesas. Embora o vento predominante no Japão tivesse origem de Oeste e devesse ter feito mais distribuição para leste sobre o Oceano Pacífico, a deposição é claramente vista a noroeste, com doses externas de aproximadamente 20-120 mSv (Sv- Sievert - unidade que representa o impacto da radiação ionizante sobre os seres humanos) e uma dose menor adicional a sudoeste com doses de aproximadamente 2-12 mSv (L. T. Dauer et al., 2011).

Estes acontecimentos despoletaram o maior evento nuclear que as autoridades japonesas declararam como nível 7, o nível mais alto da Internacional Nuclear Event Scale (INES) (L. T. Dauer et al., 2011), semelhante ao episódio de Chernobyl que ocorreu em 1986 (K. Wada, T. Yoshikawa, T. Hayashi e Y. Aizawa, 2012), (fig. 3).

Numerosos incêndios ocorreram após o terremoto, causando danos significativos, no entanto não receberam muita atenção devido aos efeitos devastadores das ondas do Tsunami. Terremotos fortes são frequentemente seguidos de incêndios e existem diversos registos no passado dessa associação (T. Tanaka, 2012).

TABELA III - Sequência de eventos em Fukushima Daiichi (de 11 a 15 de Março) em Fukushima Daiichi Nuclear Power Plant Accident: facts environmental contamination, possible biological effects and countermeasures, 2012.

TABLE III - Sequence of events in Fukushima Daiichi (11-15 March) in Fukushima Daiichi Nuclear Power Plant Accident: facts environmental contamination, possible biological effects and countermeasures, 2012.

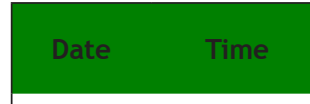

March 11 2:46 p.m.

3:42 p.m. A 14-meter tsunami triggered by the earthquake disables all AC power to Units 1, 2 and 3.

3:45 p.m. Fuel tanks for emergency diesel generators are carried off by the tsunami.

4:46 p.m. Water injection fails in the emergency core cooling systems of Units 1 and 2.

March 12 9:07 p.m. A pressure relief valve is opened on the Unit 1 pressure vessel.

3:36 p.m. A hydrogen explosion damages the structure of the Unit 1 reactor building.

8:20 p.m. Seawater injection to the Unit 1 pressure vessel begins.

5:58 a.m. Water injection fails in the emergency core cooling system of Unit 3.

March 13 9:20 a.m. A pressure relief valve is opened on the Unit 3 pressure vessel.

4:46 p.m. Water injection fails in the emergency core cooling systems of Unit 1 and 2.

11:01 a.m. A hydrogen explosion damages the external structure of the Unit 3 reactor building.

March 14 1:25 p.m.

The water level in the Unit 2 pressure vessel is found to be low, leading operators to conclude that the reactor cooling system is no longer functional.

4:34 p.m. Seawater injection into the Unit 2 pressure vessel begins.

An explosion sound is heard at Unit 2 and it concluded to indicate an abnormality in the

6:20 a.m. pressure suppression pool. At the same time, part of a wall in the operation area of Unit 4 is damaged.

9:38 a.m. A fire breaks out in the Unit 4 reactor building.

March 15 12:29 p.m. The unit 4 fire is extinguished. 


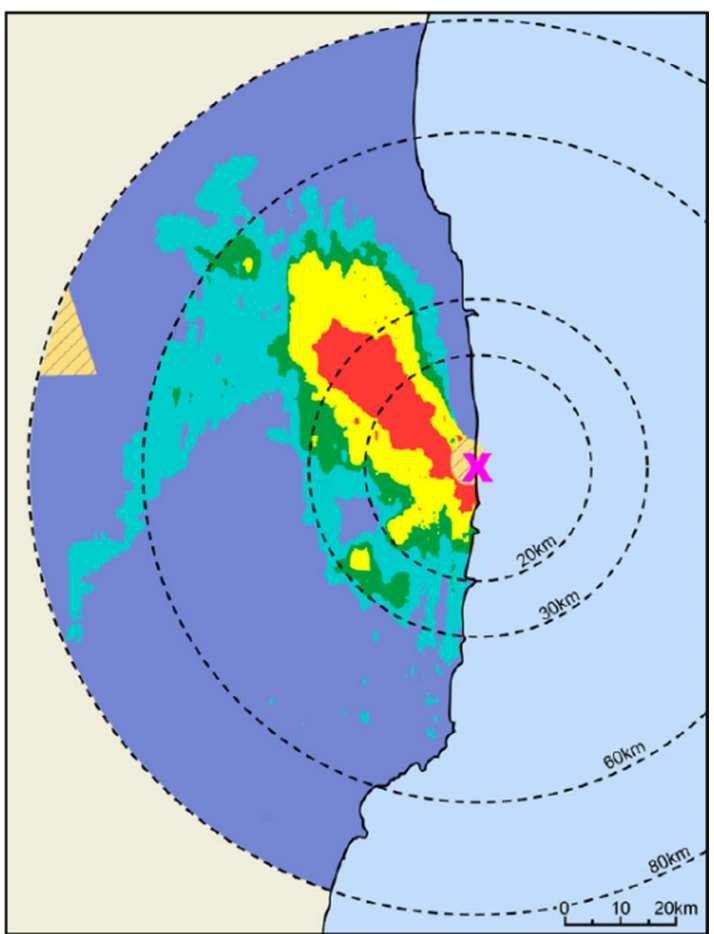

Fig. 2 - Distribuição geográfica dos produtos radioativos de fissão de césio após acidente na instalação nuclear Dai-ichi, com base no monitoramento aéreo. ( $\mathrm{X}=$ Fukushima; área tracejada $=$ sem informação; vermelho $=3-30 \mathrm{MBq} / \mathrm{m}^{2}$; amarelo $=1$ $3 \mathrm{MBq} / \mathrm{m}^{2}$; verde $=0,6-1 \mathrm{MBq} / \mathrm{m}^{2} ;$ azul esverdeado verde claro=0,3-0,6 MBq $/ \mathrm{m}^{2}$; azul escuro $=<0,3 \mathrm{MBq} / \mathrm{m}^{2}$ ), em “The Japanese Tsunami and Resulting Nuclear Emergency at the Fukushima Daiichi Power Facility: Technical, Radiologic, and Response Perspectives”, 2011.

Fig. 2 - Geographical distribution of radioactive fission products cesium after accident at the nuclear facility Dai -ichi, based on air monitoring. ( $X=$ Fukushima, dashed $=$ no information; red $=$ 3-30MBq / $\mathrm{m}^{2}$; yellow $=1-3 \mathrm{MBq} / \mathrm{m}^{2}$; Green $=0.6-1 \mathrm{MBq} / \mathrm{m}^{2}$, green green light blue $=0.3-0.6 \mathrm{MBq} / \mathrm{m}^{2}$, dark blue $=<0.3$ $\mathrm{MBq} / \mathrm{m}^{2}$ ) in "The Japanese Tsunami and Nuclear Emergency Resulting at the Fukushima Daiichi Power Facility: Technical, Radiologic, and Response Perspectives “ 2011.

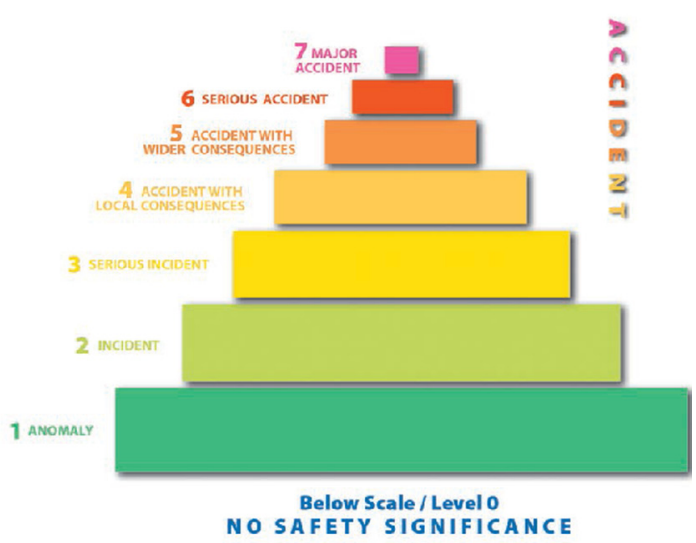

Fig. 3 - Escala Internacional de eventos nucleares e radiológicos, por International Atomic Energy Agency (IAEA).

Fig. 3 - International range of nuclear and radiological events, International Atomic Energy Agency (IAEA).
A Fire and Disaster Management Agency confirmou 15.898 mortes, 6.115 feridos e 3.917 desaparecidos, bem como 117.652 edifícios completamente destruídos e 178.200 edifícios severamente danificados em 18 localidades, os quais foram causados maioritariamente pelas ondas do Tsunami, em vez do tremor (T. Tanaka, 2012).

\section{Socorro a multivítimas}

As quantidades significativas de radioatividade lançadas no ambiente tornaram mandatórias as evacuações e medidas preventivas (L. T. Dauer et al., 2011). A prioridade inicial era minimizar o risco de nova explosão de reatores e restaurar o sistema de arrefecimento assegurando energia elétrica, que foi conseguido a 26 de Março (K. Wada et al., 2012). O governo japonês ordenou a evacuação dos habitantes ao redor da FNP-I. A zona de evacuação expandiu-se até 20 $\mathrm{km}$ a 12 de Março e dia 22 foi proibida qualquer entrada (fig. 4). Esta primeira evacuação de cerca de 20.000 pessoas só ficou completa a 15 de Março (K. Ono, 2012). A 13 de Março, iniciaram-se os protocolos de descontaminação nos centros de evacuação e a 15 de Março, estabeleceram-se os edifícios de abrigo às populações a 20-30 km da zona do acidente (L. T. Dauer et al., 2011).

Nos evacuados com menos de 40 anos foram administradas doses de iodo estável (iodeto de potássio) para proteger a tiroide da absorção de iodo radioativo (K. Ono, 2012). Tomando KI existe o risco de hipotiroidismo, que aumenta com a idade, e a capacidade de prevenir cancro da tiroide em idades superiores a 40 anos é limitada. De acordo com a Comissão de Segurança Nuclear do Japão, os trabalhadores expostos a dose equivalente de $100 \mathrm{mSv}$ para a tiroide devem tomar um comprimido de $100 \mathrm{mg} \mathrm{KI}$ no primeiro dia e $50 \mathrm{mg} \mathrm{KI}$ de seguido, todos os dias, aquando das operações. $\mathrm{O}$ limite da prescrição contínua é 14 dias (K. Wada et al., 2012).

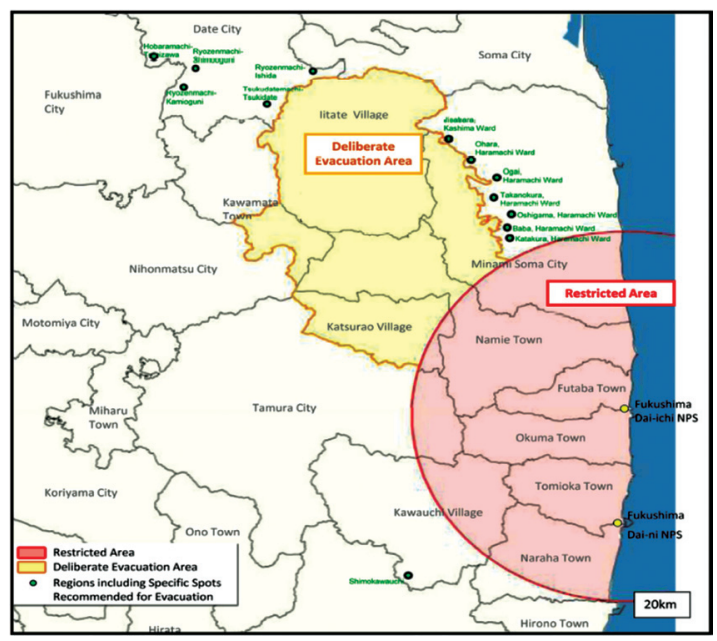

Fig. 4 - Mapa de evacuação, por Nuclear and Industrial Safety Agency (NISA), Japão. Zona rosa - Área de acesso restrito; Zona amarela - Área de evacuação deliberada.

Fig. 4 - Evacuation Map for Nuclear and Industrial Safety Agency (NISA), Japan. Pink zone - restricted area; Yellow zone - Deliberate Evacuation Area. 
Para controlar a exposição à radiação na ingestão alimentar, as autoridades japonesas instituíram medidas de controlo da água e alimentos (L. T. Dauer et al., 2011). Para minimizar a exposição externa, os níveis radiológicos foram avaliados em todo o recinto e os trabalhadores eram controlados através de dosímetro de bolso com alarme. Para proteger os trabalhadores da radiação interna através de inalação de partículas e gases radioativos, usaram máscara completa apertada com filtros P100. Para minimizar a contaminação da pele, os trabalhadores usaram macacão protetor com dupla camada de Tyvek $®$, luvas duplas (interior - algodão e exteriores - borracha) e sapatos seguros cobertos por outros de vinyl (K. Wada et al., 2012), como demonstra a foto. 1 .

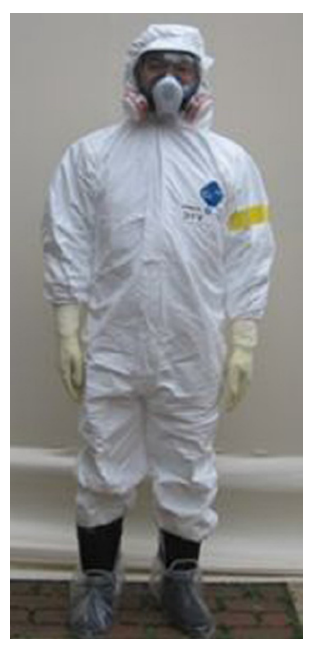

Fot. 1 - Trabalhadores da central nuclear Dai-ichi-Fukushima com macacão de proteção Tyvek® de dupla camada e um aparelho de respiração apertada com filtros P100, botas altas cobertas por sapatos de vinil, e algodão e luvas de borracha. (em Emergency response technical work at Fukushima Dai-ichi nuclear power plant: occupational health challenges posed by the nuclear disaster, 2012).

Photo. 1 - Nuclear workers Fukushima Dai -ichi - with protective overalls double layer of Tyvek® and tight breathing apparatus with P100 filters, high boots covered with vinyl shoes, and cotton and rubber gloves. (Emergency response in technical work at Fukushima nuclear power plant Dai -ichi: occupational health challenges posed by the nuclear disaster, 2012).

Apesar de alguma confusão inicial e erros de cálculo, especialmente por parte da TEPCO, relativamente à gravidade inicial da libertação de radioatividade no ambiente, foram tomadas ações protetoras pelas autoridades japonesas para diminuir a exposição da população ao iodo radioativo e césio. Situação claramente contrastante com o acidente nuclear em Chernobyl, onde as medidas protetoras não foram implementadas em tempo útil, o que resultou em grandes exposições públicas, especialmente da tiroide (L. T. Dauer et al., 2011).

\section{Triagem}

A literatura médica estabelece a origem deste conceito da palavra francesa triage, que significa escolher, classificar, selecionar (R. Noto, P. Huguernard e A. Larcan, 1994; A. J. R. Soler, L.R.J. Guadarrama e M. N. P. Corres, 2008).

O termo ambulância tem origem do latim ambulantia, o ato de caminhar. A primeira referência conhecida mostra que o conceito de ambulância terá sido idealizado pela Rainha Isabel, a Católica, sob a forma de um carro puxado por cavalos para transportar os feridos no Cerco de Málaga, em 1487 (LA. Brewer, 1986; R. Noto et al., 1994).

Em 1762, em França, passou a usar-se o termo hôpitalambulant para denominar o veículo que acompanhava as campanhas de Napoleão, e que para além do transporte de feridos prestava cuidados médicos (LA. Brewer, 1986; R. Noto et al., 1994).

Em 1792, na Campanha de Reno, o Barão Dominique Jean Larrey (1766-1842), cirurgião de Napoleão, descreveu pela primeira vez a necessidade de organizar e categorizar os primeiros socorros prestados às vítimas na frente de batalha e projetou as ambulances volantes, que puxadas por cavalos, transportavam os cirurgiões e suprimentos médicos com rapidez para o campo de batalha (F. Barot, 1998; LA. Brewer, 1986; R. Noto et al., 1994; I. Rocha, 2003; A.J.R. Soler et al., 2008).

Este foi o primeiro a separar os feridos em três grupos:

\section{Sobrevive com tratamento;}

\section{Sem grandes ferimentos esperados;}

\section{Destinado a morrer (R. Noto et al., 1994).}

Na perspetiva de R. Noto et al. (1994), triagem é um ato médico-organizacional para definir prioridade na gestão de mortes em massa, combinando a avaliação diagnóstica e prognóstica permanente com a condição formal de evacuação que poderá incluir procedimentos de reanimação e cirúrgicos baseados em requisitos técnicos, táticos e logísticos. Presentemente, no contexto clínico, a triagem consiste na separação de doentes, não em função do diagnóstico, mas sim, do prognóstico (A. M. Silva, 2009).

Sendo assim, a triagem não será um fim em si mesmo, ( $R$. Noto et al., 1994), justificando-se esta valorização por integrar o núcleo de assuntos chave na gestão da situação de exceção: comando, segurança, comunicações, avaliação, triagem, tratamento e transporte (ALSG, 1995; A. M. Silva, 2009).

Por outro lado, a triagem de catástrofe moderna tenta assegurar tanto a priorização do atendimento para os que mais necessitam como uma distribuição justa dos recursos, de modo que os profissionais de saúde consigam "fazer o máximo pela maioria" (P. Aitken e G. FitzGeral, 2012).

As características desejáveis do sistema idealizado para valorizar os pressupostos descritos são: rapidez na execução, facilidade na compreensão e implementação, ser reproduzível, ser dinâmica (evoluir com o tempo e conceitos) e, em caso de situações de catástrofe, ser capaz de prever resultados finais (ALSG, 1995; A. M. Silva, 2009). 
A necessidade de validação destes algoritmos é impulsionada pela preocupação das "sub triagem" e "sobre triagem" e pelo melhor uso dos recursos. "Sub Triagem" consta da não identificação de doentes que poderiam beneficiar de cuidados mais precoces ou diferenciados. "Sobre Triagem" origina número elevado de pacientes com categoria alta na triagem por uma hipervalorização, ultrapassando os limites dos recursos disponíveis, aumentando também a morbi e mortalidade. O algoritmo ideal garantirá cuidados primeiro aos mais necessitados, com lesões não mortais, não os desperdiçando em tentativas inúteis para salvar aqueles com lesões irrecuperáveis (P. Aitken e G. FitzGerald, 2012; A. Hirshberg, E. R. Frykberg, K. L. Mattox e M. Stein, 2010; A. J. R. Soler et al., 2008).

A classificação dos vários sistemas de triagem tem variáveis fisiológicas, anatómicas, bioquímicas, etc. Há métodos funcionais ou fisiológicos (sinais vitais, como Pressão Arterial, Frequência Respiratória e nível de consciência), que apresentam boa correlação com o prognóstico vital (A. J. R. Soler et al., 2008), sendo que a componente motora da Escala de Coma de Glasgow é a mais bem relacionada com o prognóstico do doente (S. Ross, 1998; A. M. Silva, 2009).

Devido à influência dos EUA, o sistema mais utilizado na emergência é a START Simple Triage and Rapid Treatment (F. Bozman, 2003) , sendo que esta foi desenvolvida na década de 80 , na Califórnia, pelo Hoag Memorial Hospital e pelo Newport Beach Fire Department. Esta determina rapidamente a gravidade das lesões, não requer grande capacidade de diagnóstico, integra procedimentos básicos de tratamento e estabilização e é de fácil aprendizagem (M. Silvatreger, 1998) (fig. 5). O que distingue a START é a inovação da introdução do componente motor da Escala de Coma de Glasgow (A. M. Silva, 2009).

\section{START Triage}

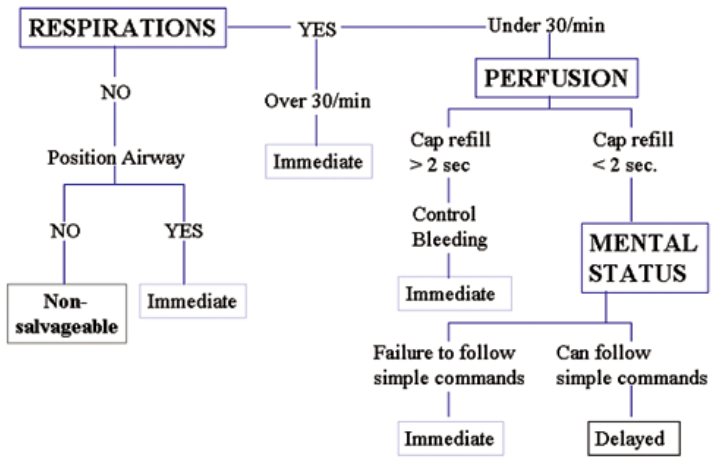

Fig. 5 - Simple Triage and Rapid Treatment (Bozman, 2003), em Triagem Prioridades - Triagem Manchester, 2009.

Fig. 5 - Simple Triage and Rapid Treatment (Bozman, 2003), in Priorities Screening - Screening Manchester, 2009.
Existem vários algoritmos adaptados para uma abordagem específica pediátrica.

O Trauma Sieve and Sort valoriza a Revised Trauma Score, sendo esta solução parte integrante da metodologia preconizada pela Triagem de Manchester nas situações de exceção (A. M. Silva, 2009).

A triagem é o primeiro passo e deve promover continuidade assistencial e para se localizar de forma espaço-temporal, segundo A. J. R. Soler et al. (2008), podemos distinguir 3 zonas específicas de triagem:

1. Zona quente (área de salvamento): no local do acidente, onde é realizada a Triagem Primária (fig. 6). Esta pretende reduzir a confusão inicial, dinamizar o processo precocemente e desocupar o local, conseguindo-se a primeira fase de contenção e organização. Um dos objetivos é não saturar o Posto Médico Avançado (PMA);

2. Zona temperada (área de socorro): só deviam chegar os de prioridade 1 e 2 . A Triagem Primária tem a sua continuidade à entrada do PMA com Triagem Secundária (fig. 7). Esta avalia FR, Pressão Arterial Sistólica (PAS) e estado de consciência (segundo Escala de Coma de Glasgow) baseada no Triage Revised Trauma Score (TRTS). 0 objetivo é determinar quem se trata primeiro no mesmo nível de prioridade. Esta deve ser contínua e, por isso, repetida sempre que houver mudança aparente de prioridade do paciente. À saída do PMA volta a realizar-se Triagem Primária para evacuação;

3. Hospitais: realiza-se triagem para hierarquizar as urgências cirúrgicas, otimizando o uso da salas operatórias, unidades de cuidados intensivos, bancos de sangue, etc.

A eficácia e a fiabilidade da triagem estão relacionadas com a perícia e experiência do elemento que tria, pelo que, em situações de catástrofe, é recomendada a presença de um médico diferenciado no posto de triagem (H. Champion, 1988; A. M. Silva, 2009).

A pontuação 12 no TRTS representa um risco de mortalidade inferior a $1 \%, 5$ de $50 \%$ e 1 de $75 \%$, ou superior (ALSG, 1995; A. M. Silva, 2009).

Atualmente, em Portugal, encontra-se em estudo, a aplicabilidade de um método de triagem simplificado, em Tese de Doutoramento em Ciências Médicas, ICBASUP "Sistema Global de Gestão Clínica, de Triagem e Evacuação Médica em eventos multivítimas aplicado a um Hospital de Campanha” (P. Campos, 2013).

Existem diferentes tipos de sistemas de identificação da prioridade relativa dos doentes. Idealmente, estes triage tags deveriam respeitar as seguintes 
características: possuir forma segura de fixação ao corpo da vítima, ser resistente às intempéries climáticas, ter superfície fácil para registo escrito, permitir o registo do nome do doente, sexo, lesões, intervenções, escalas de pontuação em uso, prioridade clínica atribuída (bem visível) e identificação do socorrista. É muito importante garantir a boa visibilidade da prioridade / cor atribuída e, se possível, deve existir mecanismo que possibilite o

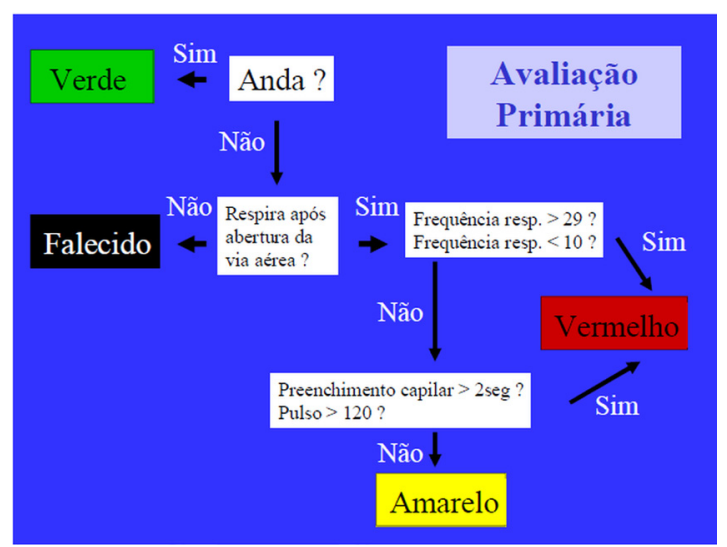

Fig. 6 - Triagem Militar em Situação de Catástrofe, em Triagem Prioridades - Triagem Manchester, 2009.

Fig. 6 - Screening military in disaster situation, In Screening Priorities - 2009 Manchester Triage. registo da passagem de uma prioridade para outra, no sentido ascendente e descendente (ALSG, 1995; A. M. Silva, 2009).

O METTAG (figs. 8 e 9) constitui o modelo mais utilizado como referência, seja para o trauma ou em situações com doentes contaminados por substâncias biológicas ou químicas (A. M. Silva, 2009).

O cruciforme Card (fot. 2), originalmente desenvolvido no Reino Unido, foi traduzido para Português e adotado no contexto do Plano de Contingência do Hospital de Santo António (A. M. Silva, 2009).

De acordo com a conferência científica nas Journee Scientifique de la SFMC da Cellule d'urgence médicopsychologique (CUMP) em 2011, as organizações de apoio psicológico presentes numa catástrofe participam na regulação da crise, ajudam na criação de uma ligação e propõem-se como conselheiro no nível médicopsicológico, podendo prevenir e tratar a natureza crónica do Transtorno de Stress Pós- Traumático (TSPT) (P. Baro e C. Pizarro, 2011).

Segundo Prof. Denis Safran, o Código Deontológico Médico diz-nos que o médico deve, em todas as circunstâncias, esforçar-se para aliviar o sofrimento do doente, através de tratamentos apropriados ao seu estado.

\section{Folha de Catástrofe}

Grupo Portuguâts de Iriage

1- Avaliação Primária

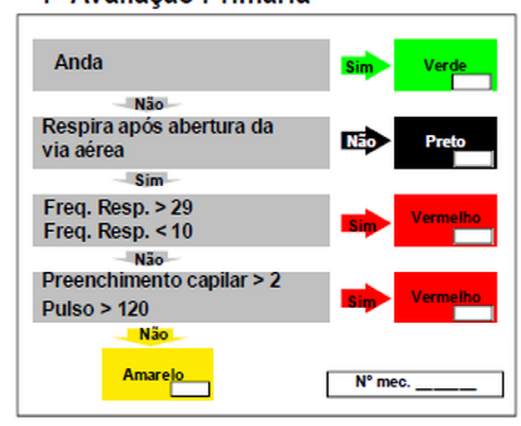

2- Avaliação Secundária

\begin{tabular}{|c|c|c|c|c|c|c|c|c|c|c|c|c|}
\hline \multicolumn{13}{|l|}{ Hora } \\
\hline & Av & TRTS & Av & TRTS & Av & TRTS & Av & TRTS & $A v$ & TRTS & Av & TRTS \\
\hline \multicolumn{13}{|l|}{ Freq.Resp. } \\
\hline \multicolumn{13}{|l|}{ P.A. Sist. } \\
\hline \multicolumn{13}{|l|}{ Glasgow } \\
\hline & $\mathbf{T}$ & & $\mathrm{T}$ & & $T$ & & $\mathbf{T}$ & & $\mathbf{T}$ & & $T$ & \\
\hline \multirow{2}{*}{\multicolumn{13}{|c|}{$\begin{array}{l}\text { Cor } \\
\text { Prioridade }\end{array}$}} \\
\hline & & & & & & & & & & & & \\
\hline $\mathrm{N}^{\circ}$ Mec. & & & & & & & & & & & & \\
\hline
\end{tabular}

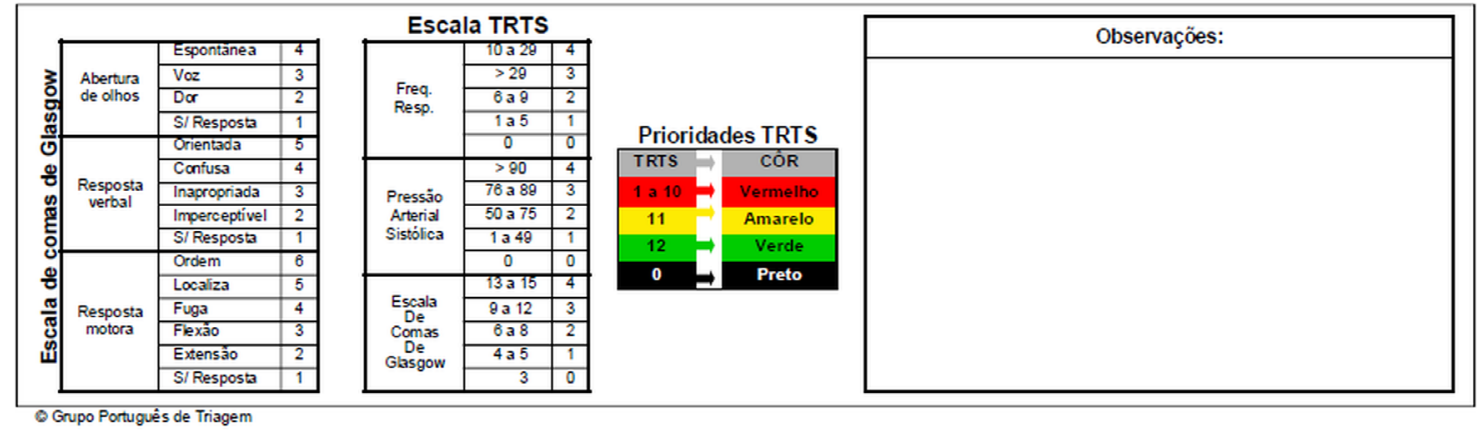

Fig. 7 - Triage Sort - Avaliação Secundária na Catástrofe, em Triagem de Manchester, em Triagem de Prioridade - Triagem de Manchester, 2009.

Fig. 7 - Triage Sort - Secondary Evaluation in Catastrophe in Manchester Triage in Priority Screening - Manchester, 2009 Screening. 
FRONT

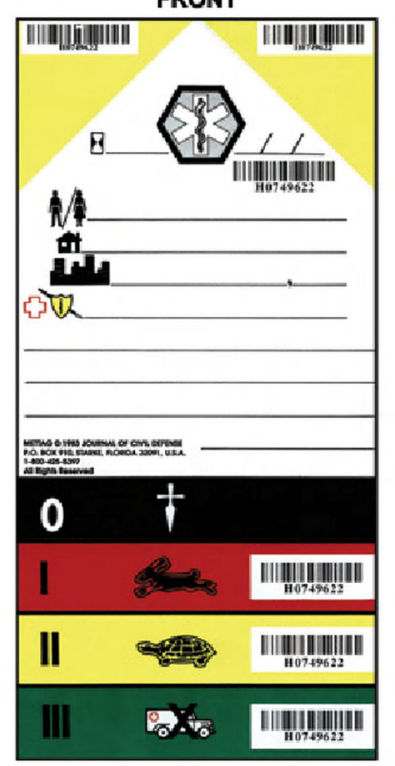

BACK

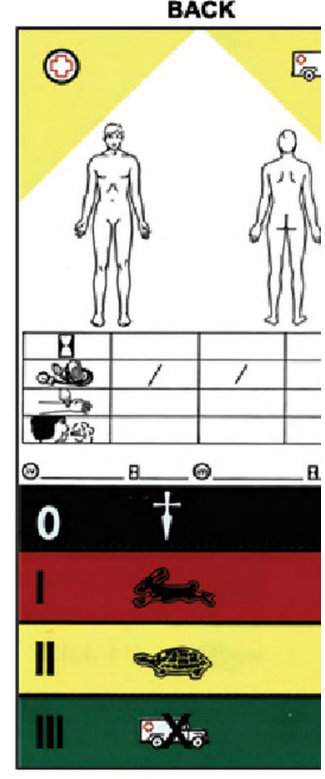

Fig. 8 - Exemplo de METTAG - Medical Emergency Triage Tag, em Triagem de Prioridades-Triagem de Manchester, 2009.

Fig. 8 - Example METTAG - Medical Emergency Triage tags, Priorities - Screening Manchester Triage, 2009.

\section{FRONT}

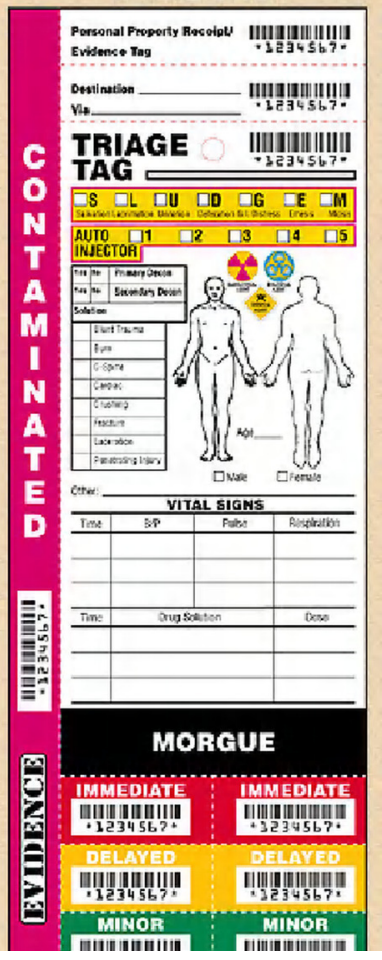

BACK

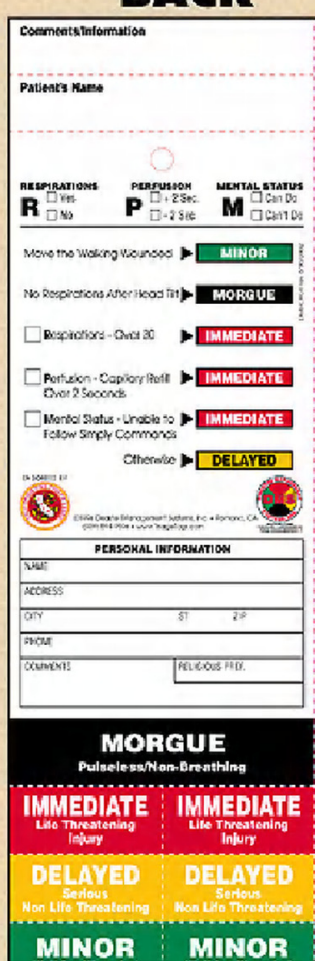

MUNOR MUNOR

Fig. 9 - Exemplo de METTAG - Medical Emergency Triage Tag com possibilidade de registar "vítimas contaminadas" e alteração de prioridade, em Triagem de Prioridades-Triagem de Manchester, 2009.

Fig. 9 - Example METTAG - Medical Emergency Triage Tag with possibility to register "contaminated victims" and changing priority in Priorities - Screening Manchester Triage, 2009.

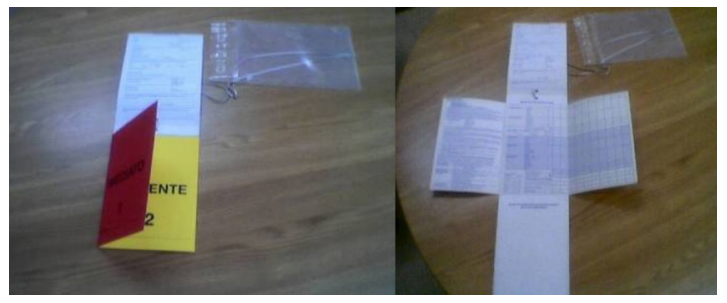

Fot. 2 - Cruciforme Card - Hospital de Santo António, em Triagem Prioridades - Triagem Manchester, 2009.

Photo. 2 - Cruciform Card - St. Anthony's Hospital in Priorities Screening - Screening Manchester, 2009.

No ambiente particularmente difícil e até hostil deste modo de exercício singular, enquanto os doentes estão frequentemente submetidos à ansiedade, à agitação e à dor, devidas não só às patologias das quais são vítimas, mas também aos gestos médicos que elas impõem, a prática da sedação, da analgesia e da anestesia reveste-se de importância primordial, sendo muitas vezes parte integrante do tratamento em situação de perigo de vida (P. Hertgen e C. Fuilla, 2006) (Tradução por R. Bandeira).

\section{Posto Médico Avançado}

Na história de Medicina de Catástrofe, o PMA surge como conceito autónomo quando o socorrista perante um cenário de multivítimas tem a necessidade de fazer a simbiose entre prestar cuidados médicos no local do sinistro ou próximo, por um lado, e por outro otimizar os meios existentes com vista a uma evacuação que de forma alguma poderia ser descontrolada (I. Rocha, 2003).

Impulsionado por Larrey e Percy, o Decreto de 21 e 27 de Abril de 1792 cria os hospitais de campanha e os hospitais ambulantes (F. Barot, 1998; I. Rocha, 2003). O conceito de PMA, tal como é atualmente interpretado, surgiu em 1985, quando Louvier e Levy criaram a noção de PMA constituído por módulos, surgindo o primeiro módulo de PMA no Alto Reno (I. Rocha, 2003).

O PMA como centro de triagem e de prestação de socorros obriga a critérios de instalação, a organização espacial adequada e a uma definição de funções (R. Bandeira, 2008; H. Julien, P. Fontaine, P. Menage e A. Lienhard, 1990). Relativamente à estrutura do PMA, deseja-se que seja uma estrutura móvel, de fácil transporte e montagem, que possa ser instalada o mais próximo possivel do local onde a situação ocorre. Preconiza-se levar para o terreno os cuidados que são prestados em ambiente hospitalar.

Uma alternativa à estrutura física cruciforme do PMA, é uma estrutura losangular (fot. 3 ), uma vez que segundo Julien 1993, fica otimizado o rendimento dinâmico do mesmo (R. Bandeira, 2008). 

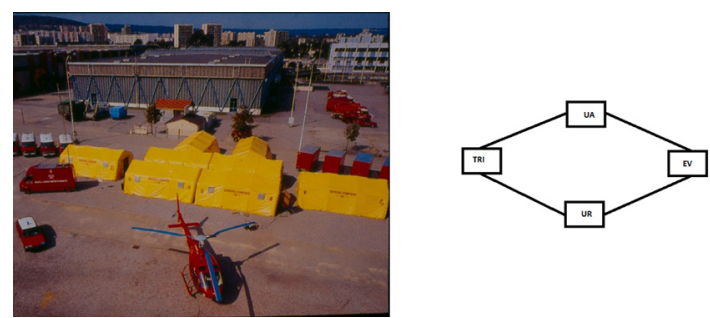

Fot. 3 - Exemplo de PMA francês com representação da estrutura losangular, UA - Urgência absoluta; UR - Urgência relativa; TRI-Triagem; EV-Evacuação, em Journee Scientifique de la SFMC, 2011.

Photo. 3 - Example of French PMA with representatives of the diamond structure, UA - absolute urgency; UR - Urgency relative; TRI-screening; EV- evacuation in Journee Scientifique de la SFMC 2011.

Atualmente, no exército português, atua-se numa outra estrutura como demonstra a foto. 4 .

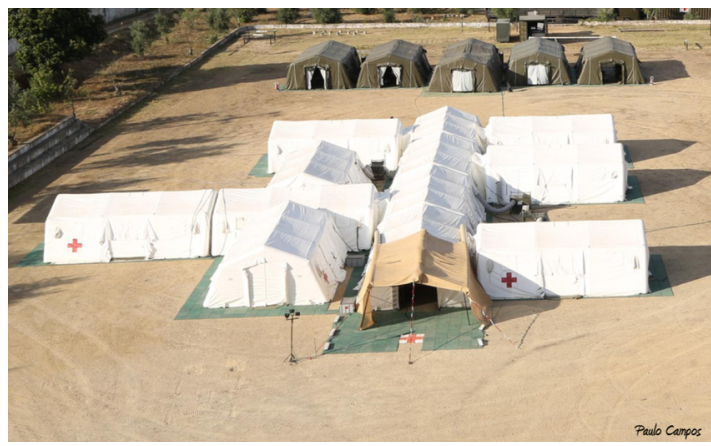

Fot. 4 - Exercício Dragão 12, em Viseu (2012), Hospital de Campanha / Role1, cedida e autorizada por Dr. Paulo Campos.

Photo. 4 - Exercise Dragon 12, Viseu ( 2012), Field Hospital / Role1, courtesy and authorized by Paulo Campos.

Quanto aos critérios de instalação, deve ter-se em conta: a segurança, a acessibilidade, a ergonomia, a proximidade do local, a direção do vento (caso haja substâncias contaminantes), proteção climática, visibilidade, a rapidez de deslocação por via terrestre e aérea (R. Bandeira, 2008; S. Briggs, 2003; A. M. Silva, 2009). Na organização espacial na área de acolhimento e triagem devem existir zonas para as emergências/ urgências, depósito mortuário, área de sujos, setor de evacuações. As funções a desempenhar pelo PMA, implicam a prestação de cuidados (reanimação e enfermagem), colocação das vítimas em condições de evacuação, apoio psicológico, abrigo dos desalojados (R. Bandeira, 2008).

Conforme Decreto-Lei $n^{\circ}$ 134/2006, de 25 de Julho, o Sistema Integrado de Operações de Proteção e Socorro (SIOPS) é o conjunto de estruturas, normas e procedimentos que asseguram que todos os agentes de proteção civil (corpos de bombeiros; forças de segurança; forças armadas; autoridade marítima e autoridade aeronáutica; INEM e demais serviços de saúde; sapadores florestais) atuam, no plano operacional, articuladamente sob um comando único, sem prejuízo da respetiva dependência hierárquica e funcional. O SIOPS visa responder a situações de iminência ou de ocorrência de acidente grave ou catástrofe (M.S. Oliveira et al., 2012).

Célula é o nome do plano do Exército Português, que apoia incidentes biológicos e químicos em território nacional, e que sistematiza as ações a desenvolver. Desde 2001 que o Exército participa no Sistema de Alerta Nacional para Incidentes NBQ (fot. 5 e 6).

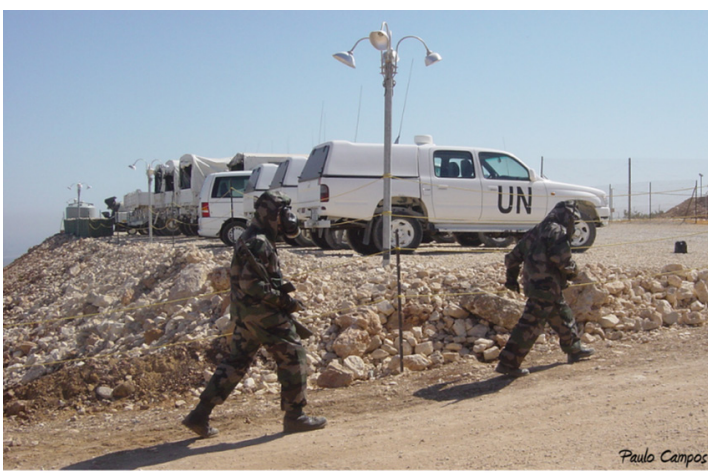

Fot. 5 - Dois operacionais com fato NBQ classe C (Libano 2007), cedida e autorizada por Dr. Paulo Campos.

Photo. 5 - Two fact operating with NBC class C (Lebanon 2007), assigned and authorized by Paulo Campos.

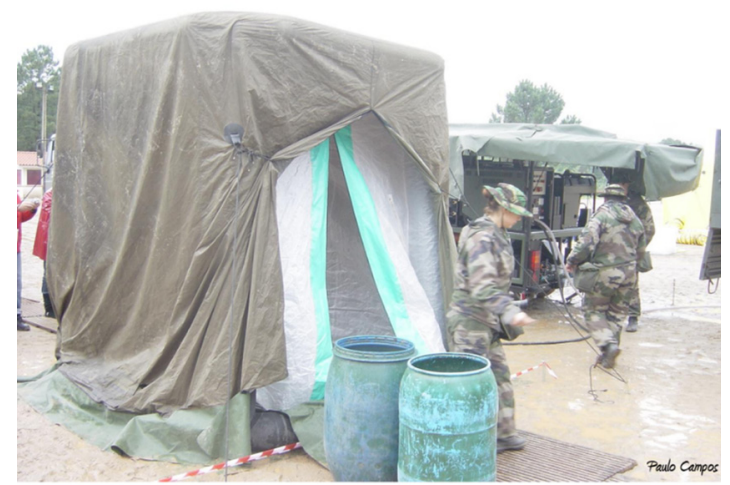

Fot. 6 - Tenda de Descontaminação, cedida e autorizada por Dr. Paulo Campos.

Photo. 6 - Decontamination Tent, courtesy and authorized by Paulo Campos.

\section{Telemedicina}

O socorro nunca é eficaz se não for apoiado por um sistema de telecomunicações fiável, com a respetiva exploração feita por equipas especialmente treinadas para o fim em vista (R. Bandeira, 2008). A Telemedicina tem aplicações potenciais em cada etapa de uma gestão de catástrofe eficaz, desde a prevenção até à preparação, resposta e recuperação (S. Simmons et al., 2008), melhorando a cobertura de serviços de emergência (K. M. Zundel, 1996). 
A primeira referência de telerradiologia é reportada a um artigo de 1950 de Gershon-Cohen descrevendo o raio-x obtido a partir de fac-similes transmitidos por fios de rádio ou telefone (K. M. Zundel, 1996). A telemedicina foi aplicada pela primeira vez em catástrofes em meados de 1980. A National Aeronautics and Space Administration (NASA) usou pela primeira vez telecomunicações para fornecer ajuda de emergência no devastador terremoto de 1985 na Cidade do México. A tecnologia avançada de comunicações por satélite foi fundamental, visto que o terremoto interrompeu todas as formas de comunicação terrestre (V. Garshnnek e F. M. Burkle, 1999).

A telemedicina engloba diagnóstico, tratamento, monitorização e educação de pacientes e providencia conveniente acesso local-independente, conselhos de especialistas e informações do paciente (V. Garshnnek e F.M. Burkle, 1999). A disponibilidade imediata da informação imagiológica aos médicos leva a uma intervenção mais precoce, melhoria do tratamento do paciente e poupança de custos (K. M. Zundel, 1996).

\section{Evacuação}

Algumas catástrofes produzem circunstâncias que exigem a remoção de emergência de alguns ou todos os cidadãos de uma área geográfica. Evacuação em massa ou de emergência pode ser dividida em evacuação imediata, onde não há aviso prévio da necessidade de evacuação, e evacuação potencial, onde é dado tempo (normalmente um ou dois dias) para evacuar. O planeamento de uma evacuação em massa frequentemente é negligenciado, no entanto deve ser planeado com detalhe. Um dado essencial é designar o responsável com autoridade para ordenar a evacuação. Os planos devem identificar prováveis cenários que exijam evacuação de emergência, os meios de comunicação à população, rotas de evacuação, os seus mecanismos (rodoviário, ferroviário, aéreo, fluvial), e providência de abrigos (R. B. Leonard, 1991).

\section{Análise Ética}

Aproximadamente 2000 a 3000 técnicos de resposta de emergência contratados pela TEPCO e empresas de suporte técnico estiveram no local do acidente diariamente até ao fim de 2011. Após 2 meses do acidente, ainda trabalhavam em condições muito precárias. Tinham de dormir no chão e alimentar-se de rações de reserva para emergências que podiam estar contaminadas (K. Ono, 2012).

A. Akabayashi e Y. Hayashi (2012), da Universidade de Graduação em Medicina de Tóquio, questionam “Terá sido eticamente justificável a evacuação obrigatória de todos os moradores com o objetivo de evitar problemas de saúde causados pela exposição à radiação?".
A primeira questão a considerar é a evidência de problemas de saúde pela exposição à radiação. Além disso, embora o risco de exposição à radiação seja o mais citado, a movimentação e evacuação também se apresentaram como sérios riscos para os idosos. Houve relatos de mortes devido às más condições nos centros de evacuação. Os riscos físicos e mentais de viver num ambiente desconhecido e sujo também são significativos (A. Akabayashi e Y. Hayashi, 2012).

O governo transportou desde 12 Março vários idosos institucionalizados para hospitais, ginásios municipais e escolas públicas. Não podiam levar valores pessoais (mesmo roupas e cobertores) devido às limitações de espaço e muitos foram transferidos várias vezes para diferentes locais durante vários meses. Evacuados tinham de se apresentar nos abrigos com certificados de "livre de radiação", pois caso não o apresentassem, eram recusados (S. Yasumura, A. Goto, S. Yamazaki e M.R. Reich, 2012).

A análise de S. Yasumura et al. (2012), na fig. 10, mostrou que a taxa de mortalidade entre os idosos institucionalizados em 2011 foi 2,4 vezes superior à de 2010. Sugere ainda que o impacto de uma catástrofe no excesso de mortalidade de idosos institucionalizados é mais significativo no rescaldo, mas tem um impacto duradouro devido às contínuas mudanças na nutrição, higiene, condições de assistência médica e gerais.

Numa outra análise é fácil criticar os médicos que se ausentam perante estas circunstâncias, no entanto a sua conduta levanta questões éticas complexas. Até que ponto são os médicos obrigados a tratar os pacientes perante um potencial colapso de uma central nuclear (A. Akabayashi, 2012)?

O princípio da beneficência dita que os médicos devem trabalhar para alcançar a saúde dos seus pacientes. Se o hospital fica dentro do raio de $20 \mathrm{~km}$, o abandono não teria consequências jurídicas pois seria considerada

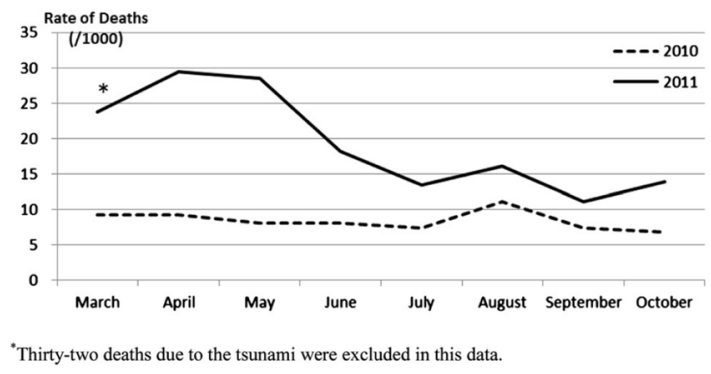

Fig. 10 - Comparação das Taxas de Mortalidade nos idosos institucionalizados entre 2010 e 2011, em “Excess mortality among relocated institutionalized elderly after the Fukushima nuclear disaster", 2012.

Fig. 10 - Comparison of mortality rates in the elderly between 2010 and 2011 in "Excess mortality Among relocated institutionalized elderly after the Fukushima nuclear disaster", 2012. 
evacuação de emergência como ordenado. No entanto, em locais a mais de $20 \mathrm{~km}$, a obrigação legal do médico de tratar os pacientes pode tornar-se um problema (A. Akabayashi, 2012).

Os códigos de Ética Médica hoje em dia chamam claramente a atenção para a obrigatoriedade de intervenção quando surja uma situação de catástrofe. 0 médico nesta situação deve assumir uma postura ética correta, na medida em que é, moral e tecnicamente, um elemento de referência comunitário (R. Bandeira, 2008).

Em Portugal desde o Código Deontológico da Associação Médica Lusitana publicado em Janeiro de 1915, passando pelo Compromisso Deontológico da Ordem dos Médicos de 1939, até ao atual Código Deontológico, está consignada a obrigatoriedade da intervenção médica em situações de urgência individual e coletiva (R. Bandeira, 2008).

\section{Consequências para a saúde a curto, médio e longo prazo}

Inicialmente, as potenciais explosões de hidrogénio e o colapso dos reatores eram os riscos mais graves. Em Março, nem todos os trabalhadores possuíam dosímetros de bolso, pois tinham sido arrastados no Tsunami. Aqueles podem também ter inalado partículas radiológicas por fuga na máscara ou por não a terem usado devido a operações de emergência inadequadas (K. Wada et al., 2012).

A partir de Maio, a exposição ao calor tornou-se um risco considerável devido à estação quente e aos fatos protetores com dupla camada Tyvek $^{\circledast}$ e respiradores de face completa que inibem o arrefecimento por evaporação. 46 trabalhadores foram notificados para consultar os médicos por sinais de insolação de Março até Outubro de 2011, não tendo havido casos fatais (K. Wada et al., 2012).

Muitos aspetos da resposta a um evento radiológico são comuns a outros eventos multivítimas, incluindo trauma, queimaduras, hemorragias, infeções, stress psicológico, num ambiente médico com condições precárias. Os eventos radiológicos têm questões adicionais de descontaminação e os efeitos da radiação no corpo como insuficiência da medula óssea, imunossupressão, queimaduras da radiação, e lesões internas de órgãos. A janela de oportunidade para contramedidas médicas é limitada (C. Norman Coleman et al., 2009).

Os agentes NRBQ entram no corpo por várias vias, variando a natureza e início dos sinais e sintomas. Gases, vapores e aerossóis, quando inalados, podem ser absorvidos por qualquer parte do trato respiratório. 0 olho também pode absorver diretamente. As gotas, e menos comummente, partículas sólidas podem ser absorvidas através da superfície da pele e membranas mucosas. Até vapores e alguns agentes voláteis podem penetrar na pele intacta e suceder intoxicação. Feridas ou abrasões são áreas mais permeáveis. Os agentes NRBQ podem contaminar comida e bebida, e assim serem absorvidos pelo trato gastrointestinal. Os tóxicos infiltrados no ambiente podem poluir os lençóis de água, o solo e o ar levando a efeitos de longo prazo dos organismos vivos (R. K. Sharma, 2010).

Podemos resumir as características de doenças infeciosas após o grande terramoto do leste do Japão em 2011, às infeções do trato respiratório (pneumonia por aspiração relacionada com o Tsunami, legionelose e influenza), infeção de feridas (tétano) e outras infeções (envenenamento de comida, doença de Tsutsugamushi e sarampo) (T. Takashi et al., 2012)

$\mathrm{Na}$ perspetiva de Lee J.K. et al., 2012, a maioria das morbi e mortalidade a curto prazo devem-se às altas doses de radiação associadas a complicações hematológicas, gastrointestinais ou cutâneas, como se viu após a catástrofe de Chernobyl.

Existem dois tipos de lesões por radiação:

\section{- Síndrome de Radiação Aguda}

Quando o corpo é exposto a altas doses de radiação, várias lesões ocorrem dependendo da dose de exposição. (K. Anzai, N. Ban, T. Ozawa e S. Tokonami, 2012)

A Síndrome de Radiação Aguda (SRA), muitas vezes chamada de Doença da Radiação, tem uma sequência de sintomas após exposição de pródromo, latência e manifestações clínicas. 0 período de latência (algumas semanas) pode preceder clínica grave como insuficiência ou falência de medula óssea (C. Norman Coleman et al., 2009).

Na catástrofe de Chernobyl, 134 funcionários da central nuclear e profissionais de emergência receberam altas doses de radiação 0.8 - 16 Gy (Gray, segundo o Sistema Internacional de Unidades de Dose de Radiação Absorvida), resultando em SRA, dos quais 28 morreram nos primeiros 4 meses. Por oposição, não existem trabalhadores com evidência de doença por SRA na catástrofe de Fukushima. Quase 99\% dos trabalhadores foram expostos a $<100 \mathrm{mSv}$ de radiação e a possibilidade de efeitos adversos futuros para a saúde é incerta. Mulheres foram proibidas de entrar na central nuclear após o acidente (K. Wada et al., 2012).

\section{- Síndrome de Radiação Tardia}

Quando o corpo é exposto a baixas doses radiológicas repetidamente alguns efeitos podem aparecer passado anos ou décadas. Entre eles, carcinogénese, como leucemia e outras malignidades, que se pensa não terem dose limiar, e catarata que tem dose limite para o início (J.K. Lee et al., 2012).

Para encontrar respostas satisfatórias sobre os efeitos agudos ou a longo termo na saúde, é necessário estimar 
as doses pessoais para os indivíduos. Para os indivíduos que não usavam equipamento anti-radiação durante o rescaldo, o dosímetro biológico (análise citogenética) era a única alternativa para avaliar as doses absorvidas. Entre os vários biomarcadores utilizados para a biodosimetria, os cromossomas dicêntricos são considerados os indicadores mais sensíveis e específicos para dosear os indivíduos recentemente sobre-expostos (J.K. Lee et al., 2012).

Apesar das limitações do estudo de J.K. Lee et al., 2012, que comparou um grupo controlo que não tinha viajado para o Japão desde o acidente nuclear com um grupo que viajou para Fukushima e consequentemente esteve exposto a níveis radiológicos (não médicos - TC ou PET), os resultados indicaram que não há atualmente nenhuma evidência de exposição entre os coreanos que viajaram para o Japão após o acidente nuclear em Fukushima.

Como demonstrado na fig. 11, as crianças geralmente têm um risco maior quando expostos a radiação (L.T. Dauer et al., 2011), sendo uma justificação possível o fato da tiroide de crianças e grávidas ser mais ativa (R.S. Groen, J.Y. Bae e K.J. Lim, 2012). O risco aumentado de cancro da tiroide, particularmente nas crianças, foi demonstrado em Chernobyl (A. Akabayashi e Y. Hayashi, 2012).

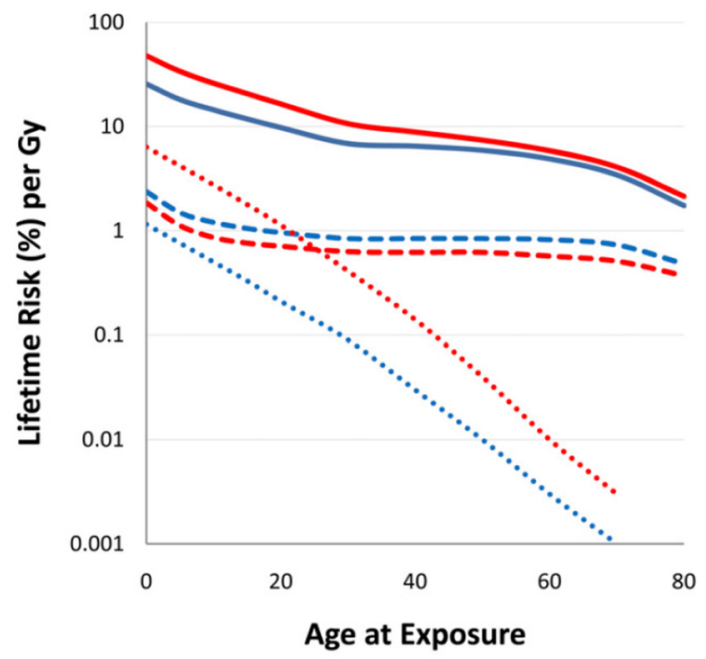

Fig. 11 - Estimativas de risco de vida atribuído à radiação com a incidência de cancro (linha contínua); cancro da tiróide (linha pontilhada); leucemia (linha tracejada) após uma exposição única baseada na idade de exposição. Homem - azul; Mulher - vermelho, em “The Japanese Tsunami and Resulting Nuclear Emergency at the Fukushima Daiichi Power Facility: Technical, Radiologic, and Response Perspectives”, 2011.

Fig. 11 - Life threatening estimates assigned to radiation with the incidence of cancer (solid line); thyroid cancer (dotted line); leukemia (dashed line) after a single exposure based on the age of exposure. Man - blue; Woman - red, "The Japanese Tsunami and Nuclear Emergency Resulting at the Fukushima Daiichi Power Facility: Technical, Radiologic, and Response Perspectives “ 2011.
Muitas grávidas estão ansiosas sobre os efeitos da radiação ionizante nos seus fetos, embora as doses pré-natais às quais foram expostos no acidente sejam inferiores às necessárias para provocar malformações (K. Ono, 2012). Os efeitos da radiação ionizante num embrião ou feto podem incluir: abortamento, malformações, anomalias neurocomportamentais, restrição do crescimento fetal e cancro. A natureza e a extensão dos efeitos determinísticos da radiação sobre a gravidez dependem da dose de radiação e do trimestre da gravidez (R.S. Groen et al., 2012). Importante também referir que doses altas de 131 l podem resultar em hipotiroidismo permanente, mas não cancro, porque atravessa a placenta e acumula-se na tiroide fetal após as 10 semanas gestacionais (S. Fushiki, 2012).

Contrariamente, a radiação pré-conceção das gónadas parentais não mostrou aumento no risco de cancro ou malformações em crianças, baseado em estudos abrangentes dos sobreviventes das bombas atómicas, bem como de pacientes tratados com radioterapia quando crianças (S. Fushiki, 2012).

O iodo estabilizado ingerido durante as refeições diárias é secretado no leite materno. A poluição ambiental com 131l é uma causa da contaminação do leite materno ( $N$. Unno et al., 2012).

Tais catástrofes podem resultar num estado de pânico geral e medo, sendo o impacto a nível físico, psicológico, económico e social. Os sobreviventes podem vivenciar reação de pânico como um efeito imediato, possibilidade de Transtorno de Stress Pós-Traumático (TSPT), síndrome de culpa do sobrevivente e outras doenças psicossociais (R. K. Sharma, 2010).

Foi realizado um estudo britânico transversal para medir várias variáveis em população de nacionalidade britânica, com pelo menos 18 anos, que estavam a habitar no Japão no momento da catástrofe. Globalmente, 46 participantes $(16,1 \%)$ apresentaram critérios para angústia, $85(29,7 \%)$ para grande ansiedade e $87(30,4 \%)$ para grande raiva (G.J. Rubin, R. Amlot, S. Wessely e N. Greenberg, 2012)

\section{Equipas de assistência}

0 recrutamento para o pessoal interventor que atua nas situações de urgência dimanadas de emergências correntes, para além dos requisitos físicos habitualmente exigidos, deve contemplar o equilíbrio psicológico dos elementos da equipa (R. Bandeira, 2008).

A Catástrofe engendra uma teia situacional de tal dimensão, em que as reais vítimas não são unicamente as pessoas primariamente atingidas; são-no por vezes também secundariamente os interventores, desde o simples contacto com os fatos até à exposição da 
própria vida; terciariamente, todos aqueles que são de algum modo afetados pelas vivências dos anteriores (R. Bandeira, 2008).

O terremoto e subsequente Tsunami, foi a pior catástrofe que o Japão vivenciou desde a Segunda Guerra Mundial, causando trauma psicológico entre os sobreviventes, bem como stress pós-incidente crítico entre as equipas de resgate. (Y. Matsuoka et al., 2011).

Um estudo anterior com profissionais que participaram nas atividades de resgate após acidente nuclear em Chernobyl revelou que a prevalência de depressão, TSPT, intenção suicida 18 anos após o acidente foi maior do que os controlos correspondentes geograficamente e que o nível de exposição à radiação estava associado com a severidade dos sintomas somáticos e de TSPT (Y. Matsuoka et al., 2012).

Foi realizado um estudo em 2012 por Matsuoka et al. a 424 membros dos 1.816 da equipa de assistência médica à catástrofe, para verificar se existe uma associação entre a preocupação subjetiva sobre a exposição à radiação e o sofrimento psicológico.

De entre todos, 9,2\% estavam preocupados com a exposição radiológica. A prevalência de doença mental grave provável e depressão, pelo Center for Epidemiologic Studies Depression Scale (CES-D) foi de $4,0 \%$ e $21,4 \%$, respetivamente, neste estudo. Para referência, a prevalência de depressão foi de 14,9\% entre os profissionais de resgate, 18 anos após o acidente de Chernobyl.

Concluindo, os resultados sugeriram que a preocupação com a exposição radiológica estava fortemente associada com as perturbações psicológicas entre os profissionais de resgate (Y. Matsuoka et al., 2012).

A experiência do acidente na FNP-I ensinou-nos que há necessidade de uma melhor formação para os profissionais médicos e paramédicos relativamente aos efeitos da radiação ionizante na saúde humana e ao sistema de proteção da radiação (K. Ono, 2012).

\section{Impacto internacional}

As libertações radioativas para a atmosfera dos reatores de FNP - I destruídos começaram a 12 de Março, 2011. Os radionuclídeos e os isótopos mais voláteis foram transportados pelo Pacífico para a América do Norte e em torno do hemisfério norte (M.K. Pham et al., 2012).

Para investigar a dispersão do material radioativo, foram coletadas e examinadas amostras de água da chuva em Berkeley, Oakland, and Albany, California. A colheita começou na noite de 15/16 de Março. Os níveis de precipitação que se observaram na San Francisco Bay não representam risco para a saúde pública (E.B. Norman, C.T. Angell e P.A. Chodash, 2011).
$\mathrm{Na}$ Europa, os primeiros sinais foram na Islândia sete dias após a fuga enquanto o primeiro pico de atividade foi observado entre 28 e 30 de Março (M.K. Pham et al., 2012). A 13 de Abril a radioatividade espalhou-se até o hemisfério sul da Ásia-Pacífico e foi detetada na Australia, Fiji, Malásia e Papua Nova Guiné (C. Potiriadis, M. Kolovou, A. Clouvas e S. Xanthos, 2012).

A análise do espectro gamma em amostras de aerossóis e precipitações detetou 131I, 134Cs e 137Cs no ar do Mónaco após o acidente em Fukushima. 0 pico de $137 \mathrm{Cs}$ observado no Mónaco foi muito menor do que o máximo observado nos acidentes de Chernobyl (2500vezes) ou de Algeciras (3vezes), como demonstrado na fig. 12.

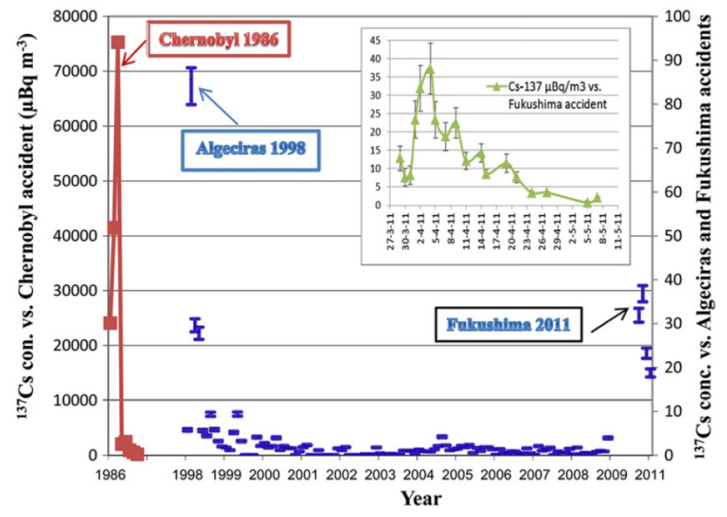

Fig. 12 - Comparação da concentração de 137Cs na atmosfera no Mónaco do acidente de Fukushima com o de Chernobyl (NOTA: valores dos eventos de Algeciras e Fukushima estão no eixo vertical direito), em "Detection of Fukushima Daiichi nuclear power plant accident radioactive traces in Monaco", 2012.

Fig. 12 - Comparison of the 137 Cs concentration in the atmosphere in Monaco Fukushima with Chernobyl . (Note: values of Algeciras and Fukushima events are on the right vertical axis) in "Detection of Fukushima Daiichi nuclear power plant accident radioactive traces in Monaco”, in 2012.

Os radioisótopos de césio e iodo estavam acima dos limites (cerca de 100 vezes o máximo), mas ainda assim sem preocupação com a exposição radiológica e saúde pública. A contaminação foi diminuindo gradualmente e as concentrações voltaram aos valores habituais após um ou dois meses (M.K. Pham et al., 2012).

Num estudo realizado por C. Potiriadis et al. em 2011, foram medidos os níveis de radioatividade ambiental nas duas maiores cidades gregas (Athens e Thessaloniki). Segundo este, 131I permaneceu na atmosfera das duas cidades por 5 semanas. A medição da concentração da atividade dos radionuclídeos artificiais na Grécia devido à fuga em Fukushima foi muito baixa, sem impacto na saúde humana. No entanto constata-se que 25 anos após o acidente de Chernobyl, vestígios de 137Cs ainda são detetados no leite de ovelha (C. Potiriadis et al., 2012). 
Situação em Portugal

Portugal, particularmente Lisboa, ficou indelevelmente marcada pelo fenómeno sísmico que ocorreu a 1 de Novembro de 1755 , tendo-se generalizado a ideia, de que só poderão ser designadas por catástrofes, as situações análogas. Esta catástrofe major e complexa tratouse de um terramoto acompanhado de maremoto e de um incêndio de proporções gigantescas que vitimaram milhares de pessoas (R. Bandeira, 2008).

Quando nos anos de 1960 e 1961 a França iniciou os seus ensaios nucleares fez detonar na atmosfera do Sahara as suas quatro primeiras bombas e parte dos detritos caiu na região costeira do Algarve, arrastados pelo vento e pela chuva, sob a forma de chuva negra (chuva radioativa). A região de Lisboa registou em 17 de Setembro 1961 com o aparecimento de chuvas Outonais e de acordo com os mesmos autores, um nível de radioatividade 41 vezes superior ao valor médio obtido no mesmo mês do ano de 1960 (R. Bandeira, 2008).

A radioatividade do acidente nuclear de FNP - I, despoletado pelo terremoto e Tsunami em Março de 2011, foi detetada na superfície aérea perto de Lisboa 14 dias após o acidente, mais especificamente 11 dias após libertação de vapores e explosões da unidade 3 a 14 de Março. Esta foi medida durante cerca de 3 semanas, mas as concentrações de atividade permaneceram baixas. A deposição de 131I, geralmente de maior preocupação devido ao impacto conhecido na saúde pública, foi baixa e de improvável contaminação significativa de gado, incluindo produção de leite e carne (F. P. Carvalho, M.C. Reis, J.M. Oliveira, M. Malta e L. Silva, 2012).

Do ponto de vista radiológico, a exposição pública à radioatividade proveniente do acidente e transportado para a região de Lisboa por processos atmosféricos foi muito baixa, sem impacto mensurável para a saúde pública (F. P. Carvalho et al., 2012).

\section{Conclusão}

Em Medicina de Catástrofe deve proceder-se a uma observação retrospetiva do Passado, que é fundamentalmente uma análise "laboratorial” histórica, no sentido de motivar a clarificação e preparação das ações no Presente, além de prevenir e predizer as mesmas para o Futuro.

Os Códigos Deontológicos ditam a obrigatoriedade da intervenção médica em situações de catástrofe. É necessário que os interventores saibam como proceder, tenham capacidade física e psicológica, elaborem um plano prático, objetivo, e rápido, com uma triagem eficaz, um espaço para prestação de cuidados médicos/ cirúrgicos imediatos e uma evacuação previamente delineada. Isto só é conseguido em contexto de simulação, com a complexidade que the é inerente, e formação subespecializada, como por exemplo, pósgraduações ou formações intra-hospitalares para todos os profissionais que lá trabalham, e que infelizmente ainda é muito escassa no nosso país. Com a telemedicina (uma área com grande margem de progressão no nosso país), a triagem, o diagnóstico e o tratamento passam a ser feitos local-independente, reforçando a noção de intervenção global.

Não se verificaram casos de SRA em Fukushima e nos estudos posteriormente apresentados não se registaram níveis internacionais preocupantes para a saúde pública, incluindo Portugal. Após este acidente foi relançado o debate internacional sobre a continuidade de centrais nucleares em funcionamento.

Enquanto país propenso a catástrofes naturais, nomeadamente terramotos, como já verificado, e a possível contaminação radiológica atmosférica de acidentes nucleares noutros países, é importante uma melhor preparação para os médicos portugueses para uma abordagem adaptada a um socorro de multivítimas, com equipamentos, meios e procedimentos específicos, diferente da Medicina de Urgência habitual.

\section{Bibliografia}

Aitken, P., \& FitzGerald, G. (2012). Disaster triage: evidence, consistency and standard practice. Emerg Med Australas, 24(3), p. 222-224. Doi:10.1111/j.1742-6723.2012.01574.x

Akabayashi, A. (2012). Must I stay? -- the obligations of physicians in proximity to the Fukushima Nuclear Power Plant. Camb Q Healthc Ethics, 21(3), p. 392-395. Doi:10.1017/S0963180112000126

Akabayashi, A., \& Hayashi, Y. (2012). Mandatory evacuation of residents during the Fukushima nuclear disaster: an ethical analysis. J Public Health (Oxf), 34(3), p. 348-351. Doi:10.1093/pubmed/fdr114

Allégre, C. (1993). As Fúrias da Terra (F. Sousa, Trans.). Lisboa.

ALSG, ADVANCED LIFE SUPPORT GROUP. (1995). Triage, Major Incident Medical Management and Suport. British Medical Journal Publishing Group, p. 113-123.

Anzai, K., Ban, N., Ozawa, T., \& Tokonami, S. (2012). Fukushima Daiichi Nuclear Power Plant accident: facts, environmental contamination, possible biological effects, and countermeasures. J Clin Biochem Nutr, 50(1), p. 2-8. Doi:10.3164/jcbn.D-11-00021 
Bandeira, R. (2008). Medicina de Catástrofe. Da Exemplificação Histórica à latroética (E. d. U. d. Porto Ed. $1^{\text {a }}$ Edição ed.).

Baro, P, \& Pizarro, C. Alegria. (2011). Triage et CUMP. Paper presented at the Journee Scientifique de la SFMC, Grenoble, França.

Barot, F. (1998). La médecine d'urgence: évolution du concept, de l'Antiquité au SAMU. Amiens: Faculte de Medicine d" Amiens, Universite de Picardie Jules Verne.

Bozman, W. (2003). Mass Casualty Incident Triage. Annals of Emergency Medicine, 41(4), p. 582-583.

Brewer, LA. (1986). Baron Dominique Jean Larrey (17661842). Father of modern military surgery, innovater, humanist. J Thorac Cardiovasc Surg, 92(6), p. 1096-1098.

Briggs, S. (2003). Triage. Advanced Disaster Medical Response Manual for Providers.

Norman Coleman C., MD, Chad Hrdina, MS, Judith L. Bader, MD, Ann Norwood, MD, Robert Hayhurst, MBA, MS, Joseph Forsha, MA, . . . Ann Knebel, RN, DNSc. (2009). Medical Response to a Radiologic/Nuclear Event: Integrated Plan From the Office of the Assistant Secretary for Preparedness and Response, Department of Health and Human Services. Annals of Emergency Medicine, 53(2).

Doi:10.1016/j.annemergmed.2007.12.021

Campos, P. (2013). Sistema Global de Gestão Clínica, de Triagem e Evacuação Médica em eventos multivítimas aplicado a um Hospital de Campanha (Doutoramento em Ciências Médicas). ICBAS - UP.

Cartwright, Julyan H. E., \& Nakamura, Hisami. (2008). Tsunami: A History of the term and of scientific understanding of the phenomenon in japanese and western culture. The Royal Society, 62(2), p. 151-166. Doi:10.1098/rsnr.2007.0038

Carvalho, F. P., Reis, M. C., Oliveira, J. M., Malta, M., \& Silva, L. (2012). Radioactivity from Fukushima nuclear accident detected in Lisbon, Portugal. J Environ Radioact, 114, p. 152-156. Doi:10.1016/j.jenvrad.2012.03.005

Champion, H. (1988). The effect of medical direction on traumatriage. Journal of Trauma, 28, p. 235-239.

Dauer, L. T., Zanzonico, P., Tuttle, R. M., Quinn, D. M., \& Strauss, H. W. (2011). The Japanese Tsunami and resulting nuclear emergency at the Fukushima Daiichi power facility: technical, radiologic, and response perspectives. J. Nucl Med, 52(9), p. 1423-1432. Doi:10.2967/jnumed.111.091413
Domingos, Alferes RC F. (2013). Um pedaço de terra defendido. Jornal do Exército, Fevereiro, 24-33.

Fushiki, S. (2012). Radiation hazards in children - Lessons from Chernobyl, Three Mile Island and Fukushima. Brain Dev. Doi:10.1016/j.braindev.2012.09.004

Garshnek, V., \& Burkle, F. M., Jr. (1999). Applications of telemedicine and telecommunications to disaster medicine: historical and future perspectives. $J$ Am Med Inform Assoc, 6(1), p. 26-37.

Groen, R. S., Bae, J. Y., \& Lim, K. J. (2012). Fear of the unknown: ionizing radiation exposure during pregnancy. Am J Obstet Gynecol, 206(6), p. 456-462. Doi:10.1016/j.ajog.2011.12.001

Hertgen, P., \& Fuilla, C. (2006). Analgesia, Sedação e Anestesia Pré-Hospitalares - Princípios e Protocolos (Bandeira R., M. Lopes \& D. Moura, Trans. U. P. Ed Ed. $1^{\text {a }}$ ed.). Porto.

Hirshberg, A., Frykberg, E.R., Mattox, K.L., \& Stein, M. (2010). Triage and trauma workload in mass casualty: a computer model. J. Trauma, 69, p. 1074-1081.

Julien, H., Fontaine, P., Menage, P., \& Lienhard, A. (1990). Caractéristiques des postes médicaux avancés de situation de catastrophe civile. Urgences Medicales, 5, p. 293-306.

Keim, Mark E. (2006). Cyclones, Tsunamis, and Human Health. The Key Role of Preparedness Oceanograph. Vol. 19, p. 40-49.

Lee, J. K., Han, E. A., Lee, S. S., Ha, W. H., Barquinero, J. F., Lee, H. R., \& Cho, M. S. (2012). Cytogenetic biodosimetry for Fukushima travelers after the nuclear power plant accident: no evidence of enhanced yield of dicentrics. J Radiat Res, 53(6), p. 876-881. Doi:10.1093/jrr/rrs065

Leonard, Ralph B. (1991). Emergency Evacuations in Disasters. Prehospital and Disaster Medicine, 6(04), p. 463-466. Doi:10.1017/S1049023X00038978

Matsuoka, Y., Nishi, D., Nakaya, N., Sone, T., Hamazaki, K., Hamazaki, T., \& Koido, Y. (2011). Attenuating posttraumatic distress with omega-3 polyunsaturated fatty acids among disaster medical assistance team members after the Great East Japan Earthquake: the APOP randomized controlled trial. BMC Psychiatry, 11, 132. Doi:10.1186/1471-244X-11-132

Matsuoka, Y., Nishi, D., Nakaya, N., Sone, T., Noguchi, H., Hamazaki, K., Koido, Y. (2012). Concern over radiation exposure and psychological distress among rescue workers following the Great 
East Japan Earthquake. BMC Public Health, 12, 249. Doi:10.1186/1471-2458-12-249

Norman, E. B., Angell, C. T., \& Chodash, P. A. (2011). Observations of fallout from the Fukushima reactor accident in San Francisco Bay area rainwater. PLoS One, 6(9), e24330. Doi:10.1371/journal.pone.0024330

Noto, R., Huguernard, P., \& Larcan, A. (1994). Medicine de Catastrophe (Masson Ed. $2^{\text {a }}$ ed. Paris ed.).

Oliveira, M. S., Meira, L., Valente, M., Catarino, R., Cunha, S., Brito, B., \& Borges, B. (2012). Manual Situação de Exceção (1 $1^{\text {a }}$ Edição ed.): Instituto Nacional de Emergência Médica.

Ono, K. (2012). Fukushima nuclear power station: what happened? Why all health care professionals need radiation training. Aesthetic Plast Surg, 36(2), p. 231-233. Doi:10.1007/s00266-011-9852-3

Pham, M. K., Eriksson, M., Levy, I., Nies, H., Osvath, I., \& Betti, M. (2012). Detection of Fukushima Daiichi nuclear power plant accident radioactive traces in Monaco. J Environ Radioact, 114, p. 131-137. Doi:10.1016/j.jenvrad.2012.01.010

Potiriadis, C., Kolovou, M., Clouvas, A., \& Xanthos, S. (2012). Environmental radioactivity measurements in Greece following the Fukushima Daichi nuclear accident. Radiat Prot Dosimetry, 150(4), p. 441-447.

Doi:10.1093/rpd/ncr423

Richter, Charles F. (1935). An instrumental earthquake magnitude scale. Bull. Seism. Soc. Am, 25(1), 1-32.

Rocha, I. (2003). Posto Médico Avançado. (Mestre), Universidade do Porto.

Ross, S. (1998). Efficacy of the Motor Component of the Glasgow Coma Scale in Trauma Triage. The Journal of Trauma: Injury, Infection and Critical Care, 45(1), p. 42-44.

Rothé, J. P. (1946). Séisme et Volcans. Paris.

Rubin, G. J., Amlot, R., Wessely, S., \& Greenberg, N. (2012). Anxiety, distress and anger among British nationals in Japan following the Fukushima nuclear accident. Br J Psychiatry, 201, p. 400407. Doi:10.1192/bjp.bp.112.111575

Sharma, R. K. (2010). Chemical, Biological, Radiological, and Nuclear disasters: Pitfalls and perils. Journal of Pharmacy and Bioallied Sciences, 2(3), 155.

Silva, A. M. (2009). Triagem Prioridades - Triagem Manchester. (Mestre), Universidade do Porto.
Simmons, S., Alverson, D., Poropatich, R., D’lorio, J., DeVany, M., \& Doarn, C. R. (2008). Applying telehealth in natural and anthropogenic disasters. Telemed J E Health, 14(9), 968-971. Doi:10.1089/tmj.2008.0117

Soler, A.J.R., Guadarrama, L.R.J., \& Corres, M.N.P. (2008). Manual de triage prehospitalario: Elsevier.

Streger, M. (1998). Prehospital Triage. Emergency Medicine Service.

Takashi, T., Mieko Goto, Haruno Yoshida, Hiroyuki Sumino, Hidenori Matsui. (2012). Infectious Diseases after the 2011 Great East Japan East Japan Earthquake. J Exp Clin Med, 4(1), p. 20-23.

Tanaka, T. . (2012). Characteristics and problems of fires following the Great East Japan earthquake in March 2011. Fire Safety Journal, 54, p. 197-202.

Ugletveit, F., \& Molhoek, W. (2004). Ongoing efforts to improve the international nuclear and radiological emergency response. Radiat Prot Dosimetry, 109(1-2), p. 149-150. Doi:10.1093/rpd/nch251

Unno, N., Minakami, H., Kubo, T., Fujimori, K., Ishiwata, I., Terada, H., Yoshimura, Y. (2012). Effect of the Fukushima nuclear power plant accident on radioiodine $((1)(3)(1)$ I) content in human breast milk. J Obstet Gynaecol Res, 38(5), 772779. Doi:10.1111/j.1447-0756.2011.01810.x

Wada, K., Yoshikawa, T., Hayashi, T., \& Aizawa, Y. (2012). Emergency response technical work at Fukushima Dai-ichi nuclear power plant: occupational health challenges posed by the nuclear disaster. Occup Environ Med, 69(8), p. 599-602. Doi:10.1136/oemed-2011-100587

Wang, Q., \& Chen, X. (2011). Nuclear accident like Fukushima unlikely in the rest of the world? Environ Sci Technol, 45(23), p. 9831-9832. Doi:10.1021/es203629u

Yasumura, S., Goto, A., Yamazaki, S., \& Reich, M. R. (2012). Excess mortality among relocated institutionalized elderly after the Fukushima nuclear disaster. Public Health. Doi:10.1016/j.puhe.2012.10.019

Zundel, Karen M. (1996). Telemedicine: history, applications, and impact on librarianship. Bulletin of the Medical Library Association, 84(1), 71. 